\title{
Matrix Optimization Over Low-Rank Spectral Sets: Stationary Points and Local and Global Minimizers
}

\author{
Xinrong Li · Naihua Xiu • Shenglong \\ Zhou
}

Received: date / Accepted: date

\begin{abstract}
In this paper, we consider matrix optimization with the variable as a matrix that is constrained into a low-rank spectral set, where the lowrank spectral set is the intersection of a low-rank set and a spectral set. Three typical spectral sets are considered, yielding three low-rank spectral sets. For each low-rank spectral set, we first calculate the projection of a given point onto this set and the formula of its normal cone, based on which the induced stationary points of matrix optimization over low-rank spectral sets are then investigated. Finally, we reveal the relationship between each stationary point and each local/global minimizer.
\end{abstract}

Keywords Matrix optimization · Low-rank spectral set · Stationary Point · Local minimizer · Global minimizer.

Mathematics Subject Classification (2000) 90C26 - 90C30 - 90C46

\section{Introduction}

In general, matrix optimization is an optimization problem, that involves matrices as variables. In this paper, we are interested in low-rank constrained matrix optimization, which aims at minimizing an objective function with a low-rank matrix as the variable. In recent years, this problem has attracted considerable attention in the fields of machine learning, signal and image processing, control, statistics and so on (see, e.g., the related surveys of $[1-5]$ ).

\footnotetext{
$\varangle$ Xinrong Li, Corresponding author

Department of Applied Mathematics, Beijing Jiaotong University, Beijing 100044, P. R. China, lixinrong0827@163.com

Naihua Xiu

Department of Applied Mathematics, Beijing Jiaotong University, Beijing 100044, P. R. China, nhxiu@bjtu.edu.cn

Shenglong Zhou

School of Mathematics, University of Southampton, Southampton, UK, sz3g14@soton.ac.uk
} 
The low-rank set (see (2) for the definition) is disjunctive and nonconvex, making the low-rank constrained matrix optimization problem NP-hard in general. Therefore, in recent decades, scholars have paid many efforts in finding different techniques to process the low-rank sets. These techniques can be summarized into three groups.

The first group consists in replacing the rank function by an approximate function. These methods include convex approximation via the nuclear norm $[6,7]$, nonconvex approximation via the Schatten $p$-norm $(0<p<1)\left[8^{-11}\right]$ and other approximation functions (see, e.g., $[12,13]$ ).

The second group consists in searching an equivalent representation for the low-rank set. In [14], Burer and Monteiro factorized a low-rank matrix as the product of two much smaller matrices. This method has also been widely studied recently, e.g., [15-19]. In [20], Gao penalized the sum of the $n-r$ smallest singular values of a matrix to force a solution to be approximately at most rank $r$, and similar approaches can be found in [21-23]. In [24], Zhou, Qi and Xiu penalized the distance between a matrix and the low-rank set, achieving a very small distance such that the matrix was almost in the lowrank set.

The third group consists in studying the low-rank constrained matrix optimization problem directly along with the point of the tangent and normal cones to the low-rank set. In [25], Luke presented the expression of the Mordukhovich normal cone (see [26, Defintion 6.4]) to the low-rank set and applied this expression to derive the local linear convergence rate of the inexact alternating projection algorithm to solve the feasibility problem (namely, finding the intersection of the low-rank set and a closed set). In [27], Cason, Absil and Van Dooren presented expressions of the Fréchet normal cone to an intersection of the low-rank set and a unit ball and proposed an iterative algorithm that converges to an $F$-stationary point of the low-rank approximation problem (see [27, Section 6$]$ ), where the Fréchet normal cone and $F$-stationary point can be found in Section 4 and (42), respectively. In [28], Schneider and Uschmajew developed a projected line-search method and established the convergence analysis via the idea of the $F$-stationary point. In [29], Zhou et al. developed a Riemannian rank-adaptive method for the low-rank constrained matrix optimization problem with an extra matrix manifold constraint. In [30], Li, Song and Xiu established the optimality conditions of low-rank constrained matrix optimization by taking advantage of the tangent and normal cones.

Motivated by the above research, this paper focuses on a class of low-rank constrained matrix optimization problems, that is, matrix optimization over low-rank spectral sets (MOLS), which aims at minimizing a general continuously differentiable objective function subject to the variable being in the intersection of a low-rank set and a spectral set. Three typical spectral sets are considered. The major contribution of this paper is to provide a comprehensive description of the stationary points and local and global minimizers of the MOLS, which establishes the first-order optimality conditions for the MOLS. Specifically, we first investigate the properties of the projection of a given point onto each low-rank spectral set and then derive the closed formulas 
of their normal cones. These formulas enable us to define two types of stationary points, namely, two kinds of first-order optimality conditions. Finally, the relationship between each stationary point and each global/local minimizer of the MOLS is revealed under some mild conditions on the objective function.

The outline of this paper is as follows. Notation definitions and basic spectral decomposition are presented in Section 2. The properties of the projections onto low-rank spectral sets are given in Section 3. Sections 4 and 5 provide closed formulas of the normal cones to low-rank spectral sets and some results of the stationary points of the MOLS. We investigate the optimality conditions associated with the stationary points and global/local minimizers in Section 6 . Finally, conclusions are presented in the last section.

\section{Preliminaries}

In this paper, we focus on the following MOLS problem

$$
\min _{X \in \mathbb{S}^{n}} f(X), \quad \text { s.t. } X \in \mathbb{S}^{n}(r) \cap \mathcal{C},
$$

where $f: \mathbb{S}^{n} \rightarrow \mathbb{R}$ is continuously differentiable with $\mathbb{S}^{n}$ denoting the space of real symmetric matrices of order $n$ equipped with the Frobenius inner product. Here, we call $\mathbb{S}^{n}(r) \cap \mathcal{C}$ a low-rank spectral set (LSS), where

$$
\mathbb{S}^{n}(r):=\left\{X \in \mathbb{S}^{n}: \operatorname{rank}(\mathrm{X}) \leq \mathrm{r}\right\}
$$

is the low-rank set with $r \in\{1,2, \cdots, n\}$ and $\operatorname{rank}(\mathrm{X})$ being the $\operatorname{rank}$ of $X$, and $\mathcal{C}$ is a spectral set in $\mathbb{S}^{n}$ (see Definition 2.1 below). Three typical choices of interest in this paper are the closed unit Frobenius ball, the symmetric box and the spectrahedron, i.e.,

$$
\begin{aligned}
& \mathcal{C}_{1}:=\left\{X \in \mathbb{S}^{n}:\|X\|_{F} \leq 1\right\}, \\
& \mathcal{C}_{2}:=\left\{X \in \mathbb{S}^{n}:-t I_{n} \preceq X \preceq t I_{n}, t>0\right\} \\
& \mathcal{C}_{3}:=\left\{X \in \mathbb{S}^{n}: X \succeq 0, \operatorname{Tr}(X)=1\right\},
\end{aligned}
$$

where $\|X\|_{F}$ is the Frobenius norm and $\operatorname{Tr}(X)$ is the trace of $X$.

Our research on MOLS is motivated by a number of important applications in various areas. For example, the graph similarity problem in $[31,27]$ is a special case of $\mathcal{C}=\mathcal{C}_{1}$. When $\mathcal{C}=\mathcal{C}_{2}$ in (1), the application covers the wireless sensor network localization problem $[32,33]$ and the nearest low-rank correlation matrix problem $[34,35]$. Moreover, the quantum-state tomography problem $[36,37]$ can be modeled by $(1)$ with $\mathcal{C}=\mathcal{C}_{3}$.

Some related fundamental notation used throughout the paper is defined as follows. Let $\mathbb{R}^{n}$ be the Euclidean space equipped with the norm $\|\cdot\|_{2}$. Let $\mathbb{S}^{n}$ be the Euclidean space of the real symmetric matrices equipped with inner product $\langle X, Y\rangle=\operatorname{Tr}(X Y)$ and the induced Frobenius norm $\|X\|_{F}:=$ $\sqrt{\operatorname{Tr}(X X)}$ for $X, Y \in \mathbb{S}^{n}$. Let $\operatorname{rank}(\mathrm{X})$ denote the rank of $X \in \mathbb{S}^{n}$. For a given vector $x \in \mathbb{R}^{n}$ and an index set $T \subseteq\{1, \cdots, n\}$, we denote $x_{T}=\left(x_{i}\right)_{i \in T} \in \mathbb{R}^{|T|}$ 
as the subvector of $x$ corresponding to the indices in $T$, where $|T|$ is the cardinality of set $T$. Similarly, $X_{T}$ is the submatrix consisting of the columns of $X$ indexed by $T$. The complementary set of $T$ is written as $T^{\perp}=\{1, \cdots, n\} \backslash T$. Define a sparse set by $\mathbb{R}^{n}(r):=\left\{x \in \mathbb{R}^{n}:\|x\|_{0} \leq r\right\}$, where $r \in\{1, \cdots, n\}$ and $\|\cdot\|_{0}$ is the $l_{0}$ norm counting the number of nonzero entries of $x$. For a vector $x \in \mathbb{R}^{n}$, let $\operatorname{Diag}(x)$ be an $n \times n$ diagonal matrix with diagonal entries $x_{i}$. Denote $\mathbb{S}_{+}^{n}$ as the space containing all the positive semidefinite matrices and $\mathbb{S}_{-}^{n}:=-\mathbb{S}_{+}^{n}$. The $n$-order identity matrix is written as $I_{n}$.

For any given matrix $X \in \mathbb{S}^{n}$ with $s:=\operatorname{rank}(X)<n$, denote

$$
\Lambda(X):=\operatorname{Diag}(\lambda(X)) \text { and } \lambda(X):=\left(\lambda_{1}(X), \cdots, \lambda_{n}(X)\right)^{\top}
$$

as the vector containing all the eigenvalues of $X$ arranged in nonincreasing order, i.e.,

$$
\lambda_{1}(X) \geq \cdots \geq \lambda_{n}(X)
$$

For a subset $\boldsymbol{\alpha} \subseteq\{1, \cdots, n\}$, write

$$
\lambda_{\boldsymbol{\alpha}}(X):=(\lambda(X))_{\boldsymbol{\alpha}}=\left(\lambda_{i}(X)\right)_{i \in \boldsymbol{\alpha}}, \quad \Lambda_{\boldsymbol{\alpha}}(X):=\operatorname{Diag}\left(\lambda_{\boldsymbol{\alpha}}(X)\right) .
$$

Let $\Gamma$ be the index set that contains all the indices of the nonzero elements of $\lambda(X)$. We then have the following eigenvalue decomposition (EVD)

$$
X=U \Lambda(X) U^{\top}=\left[\begin{array}{ll}
U_{\Gamma} & U_{\Gamma^{\perp}}
\end{array}\right]\left[\begin{array}{rr}
\Lambda_{\Gamma}(X) & \\
& 0
\end{array}\right]\left[\begin{array}{ll}
U_{\Gamma} & U_{\Gamma^{\perp}}
\end{array}\right]^{\top}=U_{\Gamma} \Lambda_{\Gamma}(X) U_{\Gamma}^{\top},
$$

where $U:=\left[U_{\Gamma} U_{\Gamma^{\perp}}\right] \in \mathcal{O}^{n}$ and $U_{\Gamma} \in \mathbb{R}^{n \times s}$ is the submatrix that contains all columns indexed on $\Gamma$ of $U$ and $\mathcal{O}^{n}$ is the set of all $n \times n$ orthogonal matrices, i.e.,

$$
\mathcal{O}^{n}=\left\{A \in \mathbb{R}^{n \times n}: A^{\top} A=A A^{\top}=I_{n}\right\} .
$$

Hereafter, for simplicity, we write the block diagonal matrix as

$$
\left[\begin{array}{ccc}
A_{1} & & \\
& \ddots & \\
& & A_{n}
\end{array}\right]:=\left[\begin{array}{ccc}
A_{1} & \cdots & 0 \\
\vdots & \ddots & \vdots \\
0 & \cdots & A_{n}
\end{array}\right],
$$

where $A_{i}$ is the matrix for $i \in\{1, \cdots, n\}$.

Definition 2.1 [38, Proposittion 5.1] The set $\mathcal{C}$ in $\mathbb{S}^{n}$ is a spectral set if there exists a symmetric set $\mathcal{K}^{1}$.) in $\mathbb{R}^{n}$ such that

$$
\mathcal{C}:=\lambda^{-1}(\mathcal{K})=\left\{X \in \mathbb{S}^{n}: \lambda(X) \in \mathcal{K}\right\}
$$

\footnotetext{
1 A set $\mathcal{K} \in \mathbb{R}^{n}$ is said to be symmetric if $P x \in \mathcal{K}$ for every $x \in \mathcal{K}$ and every $P \in \mathbb{P}^{n}$ where $\mathbb{P}^{n}$ denotes the set of all $n \times n$ permutation matrices (For those matrices that have only one nonzero entry in every row and column, which is 1 , see [38]
} 
For example,

$$
\begin{array}{ll}
\mathcal{C}_{1}=\lambda^{-1}\left(\mathcal{K}_{1}\right), & \mathcal{K}_{1}:=\left\{x \in \mathbb{R}^{n}:\|x\|_{2} \leq 1\right\}, \\
\mathcal{C}_{2}=\lambda^{-1}\left(\mathcal{K}_{2}\right), & \mathcal{K}_{2}:=\left\{x \in \mathbb{R}^{n}:\|x\|_{\infty} \leq t, t>0\right\} \\
\mathcal{C}_{3}=\lambda^{-1}\left(\mathcal{K}_{3}\right), & \mathcal{K}_{3}:=\left\{x \in \mathbb{R}^{n}:\|x\|_{1}=1, x_{i} \geq 0, i=1, \ldots, n\right\} .
\end{array}
$$

In fact, since $X=U \Lambda(X) U^{\top}=U \operatorname{Diag}(\lambda(X)) U^{\top},\|X\|_{F} \leq 1$ is equivalent to $\|\lambda(X)\|_{2} \leq 1$, which means $\lambda(X) \in \mathcal{K}_{1}$. Moreover, it is easy to find that $\mathcal{K}_{1}$ is symmetric. Then, $\mathcal{C}_{1}$ is a spectral set, and $\mathcal{C}_{1}=\lambda^{-1}\left(\mathcal{K}_{1}\right)$. Similar reasoning can be applied to derive the last two equations. In addition, one can verify that $\mathbb{S}^{n}(r)$ and $\mathbb{S}^{n}(r) \cap \mathcal{C}_{i}, i=1,2,3$ are also spectral sets, namely,

$$
\lambda^{-1}\left(\mathbb{R}^{n}(r)\right)=\mathbb{S}^{n}(r), \quad \lambda^{-1}\left(\mathbb{R}^{n}(r) \cap \mathcal{K}_{i}\right)=\mathbb{S}^{n}(r) \cap \mathcal{C}_{i}, \quad i=1,2,3 .
$$

\section{Projections}

To study the projections onto $\mathbb{S}^{n}(r) \cap \mathcal{C}$, we first introduce the metric projection, which is defined as

$$
\Pi_{\Omega}(X) \in \Pi_{\Omega}(X):=\operatorname{argmin}_{Y \in \Omega}\|Y-X\|_{F} .
$$

Here, we denote $\boldsymbol{\Pi}_{\Omega}(X)$ as the set containing all projections of $X$ onto $\Omega$, and $\Pi_{\Omega}(X)$ is a particular projection in $\Pi_{\Omega}(X) . \Pi_{\Omega}(X)$ is a singleton if $\Omega$ is a closed and convex set. The following result associated with projection onto the low-rank set is borrowed from [20, Lemma 2.9]. Let

$$
\begin{aligned}
\boldsymbol{\alpha} & :=\left\{i:\left|\lambda_{i}(X)\right|>\lambda_{r}^{\mathrm{abs}}(X)\right\}, \\
\boldsymbol{\beta} & :=\left\{i:\left|\lambda_{i}(X)\right|=\lambda_{r}^{\mathrm{abs}}(X)\right\}, \\
\boldsymbol{\gamma}: & =\left\{i:\left|\lambda_{i}(X)\right|<\lambda_{r}^{\mathrm{abs}}(X)\right\},
\end{aligned}
$$

where $\lambda_{r}^{\text {abs }}(\cdot)$ is the $r$ th largest (in absolute terms) entry of $\left(\left|\lambda_{1}(\cdot)\right|, \cdots,\left|\lambda_{n}(\cdot)\right|\right)$. Clearly, (8) implies $|\boldsymbol{\alpha}|<r$. Define

$$
\begin{aligned}
V_{Q} & :=\left[\begin{array}{lll}
U_{\boldsymbol{\alpha}} & U_{\boldsymbol{\beta}} Q & U_{\boldsymbol{\gamma}}
\end{array}\right], \\
Q & :=\left[\begin{array}{ll}
Q_{+} & \\
& Q_{-}
\end{array}\right], Q_{+} \in \mathcal{O}^{\left|\boldsymbol{\beta}_{+}\right|}, Q_{-} \in \mathcal{O}^{\left|\boldsymbol{\beta}_{-}\right|},
\end{aligned}
$$

where

$$
\boldsymbol{\beta}_{+}:=\left\{i: \lambda_{i}(X)=\lambda_{r}^{\mathrm{abs}}(X)\right\}, \quad \boldsymbol{\beta}_{-}:=\left\{i: \lambda_{i}(X)=-\lambda_{r}^{\mathrm{abs}}(X)\right\} .
$$

It is easy to check that

$$
Q \in \mathcal{O}^{|\boldsymbol{\beta}|}, \quad V_{Q} \in \mathcal{O}^{n}, \quad \Lambda_{\boldsymbol{\beta}}(X)=Q \Lambda_{\boldsymbol{\beta}}(X) Q^{\top} .
$$

We now rewrite the EVD (6) as

$$
X=\left[\begin{array}{lll}
U_{\boldsymbol{\alpha}} & U_{\boldsymbol{\beta}} & U_{\boldsymbol{\gamma}}
\end{array}\right]\left[\begin{array}{lll}
\Lambda_{\boldsymbol{\alpha}}(X) & & \\
& \Lambda_{\boldsymbol{\beta}}(X) & \\
& & \Lambda_{\boldsymbol{\gamma}}(X)
\end{array}\right]\left[\begin{array}{lll}
U_{\boldsymbol{\alpha}} & U_{\boldsymbol{\beta}} & U_{\boldsymbol{\gamma}}
\end{array}\right]^{\top},
$$


and the metric projection over the set $\mathbb{S}^{n}(r)$ is given by

$$
\Pi_{\mathbb{S}^{n}(r)}(X)=\left\{V_{Q} \operatorname{Diag}(y) V_{Q}^{\top}: \text { any } y \in \mathcal{Y} \text { and any } Q \text { satisfies }(10)\right\},
$$

where

$\mathcal{Y}=\left\{y \in \mathbb{R}^{n}: y_{i}=\left\{\begin{array}{cc}\lambda_{i}(X), & i \in \boldsymbol{\alpha} \cup \boldsymbol{\beta}_{0} \\ 0, & \text { otherwise }\end{array}\right.\right.$ with any $\left.\boldsymbol{\beta}_{0} \subseteq \boldsymbol{\beta},\left|\boldsymbol{\beta}_{0}\right|=\mathrm{r}-|\boldsymbol{\alpha}|\right\}$.

In general, the set $\Pi_{\mathbb{S}^{n}(r)}(X)$ is not singleton. The multiplicity of projection occurs only at the index set of $\boldsymbol{\beta}$. If $\operatorname{rank}(X)=s<r$, then $\lambda_{r}^{\text {abs }}(X)=0$ and $|\boldsymbol{\alpha}|=s$. In this case, $\boldsymbol{\Pi}_{\mathbb{S}^{n}(r)}(X)=\{X\}$.

We aim at calculating projections over three low-rank spectral sets to prepare for the first-order optimality conditions of problem (1) in Section 5. Before the main results are presented, we introduce lemma [39, Fact 2.3], which plays an important role in the subsequent proof.

Lemma 3.1 [39, Fact 2.3] Let $\mathcal{K}$ be a symmetric set. For any $X \in \mathbb{S}^{n}$, the projection of $X$ onto the spectral set $\lambda^{-1}(\mathcal{K})$ is given by

$$
\boldsymbol{\Pi}_{\lambda^{-1}(\mathcal{K})}(X)=\left\{U \operatorname{Diag}(y) U^{\top}: y \in \boldsymbol{\Pi}_{\mathcal{K}}(\lambda(X)), U \in \mathcal{O}^{n}(X)\right\},
$$

where the set $\mathcal{O}^{n}(X):=\left\{U \in \mathcal{O}^{n}: X=U \operatorname{Diag}(\lambda(X)) U^{\top}\right\}$.

This lemma is actually a generalization of [40, Corollary2.8] due to (7). For simplicity, write $\Pi_{\mathcal{C}} \Pi_{\mathbb{S}^{n}(r)}(X)=\Pi_{\mathcal{C}}\left(\Pi_{\mathbb{S}^{n}(r)}(X)\right)$ and

$$
\Pi_{\mathcal{C}} \Pi_{\mathbb{S}^{n}(r)}(X):=\left\{\Pi_{\mathcal{C}} \Pi_{\mathbb{S}^{n}(r)}(X): \forall \Pi_{\mathbb{S}^{n}(r)}(X) \in \Pi_{\mathbb{S}^{n}(r)}(X)\right\} .
$$

Theorem 3.1 Let $X \in \mathbb{S}^{n}$ have the EVD as in (12) and let $V_{Q}$ be given as (9). For a set $T$, by defining $\Xi_{\mathcal{C}_{1}}^{T}(X): \mathbb{S}^{n} \rightarrow \mathbb{R}^{n}$ as

$$
\left[\Xi_{\mathcal{C}_{1}}^{T}(X)\right]_{i}=\left\{\begin{array}{c}
c_{1} \lambda_{i}(X), i \in T, \\
0, \quad \text { otherwise }
\end{array}\right.
$$

where $c_{1}:=\min \left\{1, c_{F}^{-1 / 2}\right\}$ with $c_{F}:=\left\|\lambda_{\boldsymbol{\alpha}}(X)\right\|_{2}^{2}+(r-|\boldsymbol{\alpha}|)\left(\lambda_{r}^{\operatorname{abs}}(X)\right)^{2}$, it follows that

$$
\begin{aligned}
& \Pi_{\mathbb{S}^{n}(r) \cap \mathcal{C}_{1}}(X)=\Pi_{\mathcal{C}_{1}} \Pi_{\mathbb{S}^{n}(r)}(X)
\end{aligned}
$$

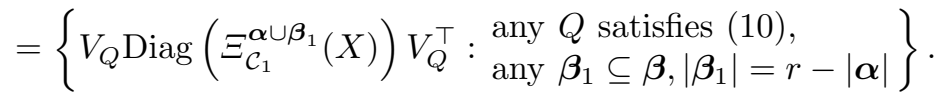

Proof. We first prove the second equation (17). For any $Q$ satisfying (10) and any $\boldsymbol{\beta}_{1} \subseteq \boldsymbol{\beta},\left|\boldsymbol{\beta}_{1}\right|=r-|\boldsymbol{\alpha}|$, every element $\Pi_{\mathbb{S}^{n}(r)}(X)$ in $\boldsymbol{\Pi}_{\mathbb{S}^{n}(r)}(X)$ from (13) takes the form

$$
\Pi_{\mathbb{S}^{n}(r)}(X)=V_{Q} \operatorname{Diag}\left(\left[\lambda_{\boldsymbol{\alpha} \cup \boldsymbol{\beta}_{1}}(X)^{\top} 0\right]^{\top}\right) V_{Q}^{\top} .
$$


Since $V_{Q} \in \mathcal{O}^{n}$ due to (11), $\boldsymbol{\beta}_{1} \subseteq \boldsymbol{\beta}$ and the definition of $\boldsymbol{\beta}$ in (8), we have

$$
\begin{aligned}
\left\|\Pi_{\mathbb{S}^{n}(r)}(X)\right\|_{F}^{2} & =\left\|\lambda_{\boldsymbol{\alpha} \cup \boldsymbol{\beta}_{1}}(X)\right\|_{2}^{2} \\
& =\left\|\lambda_{\boldsymbol{\alpha}}(X)\right\|_{2}^{2}+(r-|\boldsymbol{\alpha}|)\left(\lambda_{r}^{\mathrm{abs}}(X)\right)^{2}=c_{F} .
\end{aligned}
$$

Lemma 3.1 with $\lambda^{-1}(\mathcal{K})=\mathcal{C}_{1}$ and $X=\Pi_{\mathbb{S}^{n}(r)}(X)$ indicates

$$
\Pi_{\mathcal{C}_{1}}\left(\Pi_{\mathbb{S}^{n}(r)}(X)\right)=V_{Q} \operatorname{Diag}\left(x^{*}\right) V_{Q}^{\top},
$$

where

$$
x^{*}=\operatorname{argmin}_{\|z\|_{2} \leq 1}\left\|z-\left[\lambda_{\boldsymbol{\alpha} \cup \boldsymbol{\beta}_{1}}(X)^{\top} 0\right]^{\top}\right\|_{2} \stackrel{(18)}{=} \Xi_{\mathcal{C}_{1}}^{\boldsymbol{\alpha} \cup \boldsymbol{\beta}_{1}}(X),
$$

which verifies (17). Next, we prove (16) by proving two parts. In the first part, $\Pi_{\mathbb{S}^{n}(r) \cap \mathcal{C}_{1}}(X) \subseteq \Pi_{\mathcal{C}_{1}} \Pi_{\mathbb{S}^{n}(r)}(X)$ is proven. In the second part, we show the reverse inclusion.

Part 1. For any $\Pi_{\mathbb{S}^{n}(r) \cap \mathcal{C}_{1}}(X) \in \Pi_{\mathbb{S}^{n}(r) \cap \mathcal{C}_{1}}(X)$, Lemma 3.1, in which $\lambda^{-1}(\mathcal{K})=\mathbb{S}^{n}(r) \cap \mathcal{C}_{1}$, indicates

$$
\Pi_{\mathbb{S}^{n}(r) \cap \mathcal{C}_{1}}(X)=U \operatorname{Diag}\left(x^{*}\right) U^{\top},
$$

where $x^{*}$ is an optimal solution of

$$
\min \left\{\|z-\lambda(X)\|_{2}: z \in \mathcal{K}_{1} \cap \mathbb{R}^{n}(r)\right\} .
$$

The above problem is equivalent to

$$
\min _{z}\left\{\left\|z_{\boldsymbol{\alpha} \cup \boldsymbol{\beta}_{1}}-\lambda_{\boldsymbol{\alpha} \cup \boldsymbol{\beta}_{1}}(X)\right\|_{2}: \begin{array}{l}
\left\|z_{\boldsymbol{\alpha} \cup \boldsymbol{\beta}_{1}}\right\|_{2} \leq 1, \\
z_{i}=0, i \notin \boldsymbol{\alpha} \cup \boldsymbol{\beta}_{1}
\end{array}\right\}
$$

for any $\boldsymbol{\beta}_{1} \subseteq \boldsymbol{\beta},\left|\boldsymbol{\beta}_{1}\right|=r-|\boldsymbol{\alpha}|$ owing to [41, Lemma 1], where $\boldsymbol{\alpha}, \boldsymbol{\beta}$ are defined as in (8). Therefore, there exists one $\boldsymbol{\beta}_{1}^{\prime}$ such that $x^{*}$ is an optimal solution of (20) with $\boldsymbol{\beta}_{1}=\boldsymbol{\beta}_{1}^{\prime}$. For such $\boldsymbol{\beta}_{1}^{\prime}$, by (13) with $Q=I$, there must be one $\Pi_{\mathbb{S}^{n}(r)}(X)$ satisfying

$$
\Pi_{\mathbb{S}^{n}(r)}(X)=U \operatorname{Diag}\left(\left[\lambda_{\boldsymbol{\alpha} \cup \boldsymbol{\beta}_{1}^{\prime}}(X)^{\top} 0\right]^{\top}\right) U^{\top},
$$

which again with Lemma 3.1 with $\lambda^{-1}(\mathcal{K})=\mathcal{C}_{1}$ and $X=\Pi_{\mathbb{S}^{n}(r)}(X)$ implies

$$
\Pi_{\mathcal{C}_{1}} \Pi_{\mathbb{S}^{n}(r)}(X)=U \operatorname{Diag}\left(x_{S}^{*}\right) U^{\top}
$$

where $x_{S}^{*}$ is an optimal solution of

$$
\min _{z}\left\{\left\|z-\left[\lambda_{\boldsymbol{\alpha} \cup \boldsymbol{\beta}_{1}^{\prime}}(X)^{\top} 0\right]^{\top}\right\|_{2}:\|z\|_{2} \leq 1\right\} .
$$

The solution of the above problem is actually the same as (20) with $\boldsymbol{\beta}_{1}=\boldsymbol{\beta}_{1}^{\prime}$. Then, $x^{*}=x_{S}^{*}$, which suffices to show that $\Pi_{\mathbb{S}^{n}(r) \cap \mathcal{C}_{1}}(X) \subseteq \Pi_{\mathcal{C}_{1}} \Pi_{\mathbb{S}^{n}(r)}(X)$. 
Part 2. To show $\Pi_{\mathbb{S}^{n}(r) \cap \mathcal{C}_{1}}(X) \supseteq \Pi_{\mathcal{C}_{1}} \Pi_{\mathbb{S}^{n}(r)}(X)$, we first specify a minimizer $Y^{*} \in \operatorname{argmin}_{Y \in \mathbb{S}^{n}(r) \cap \mathcal{C}_{1}}\|Y-X\|_{F}^{2}=\Pi_{\mathbb{S}^{n}(r) \cap \mathcal{C}_{1}}(X)$ and then prove that for each $X^{*} \in \Pi_{\mathcal{C}_{1}} \Pi_{\mathbb{S}^{n}(r)}(X)$, it follows that

$$
X^{*} \in \mathbb{S}^{n}(r) \cap \mathcal{C}_{1}, \quad\left\|X-X^{*}\right\|_{F}=\left\|X-Y^{*}\right\|_{F} .
$$

Thus, $X^{*} \in \boldsymbol{\Pi}_{\mathbb{S}^{n}(r) \cap \mathcal{C}_{1}}(X)$. From the proof of Part 1, we can see that for the above, given $\boldsymbol{\beta}_{1}^{\prime}$,

$$
Y^{*}:=U\left[\begin{array}{lll}
c_{1} \Lambda_{\boldsymbol{\alpha}}(X) & & \\
& c_{1} \Lambda_{\boldsymbol{\beta}_{1}^{\prime}}(X) & \\
& & 0
\end{array}\right] U^{\top} \in \Pi_{\mathcal{C}_{1}} \Pi_{\mathbb{S}^{n}(r)}(X)
$$

also satisfies $Y^{*} \in \Pi_{\mathbb{S}^{n}(r) \cap c_{1}}(X)$. So the optimal objective function value is

$$
\begin{aligned}
f^{*} & =\min _{Y \in \mathbb{S}^{n}(r) \cap \mathcal{C}_{1}}\|Y-X\|_{F}^{2}=\left\|Y^{*}-X\right\|_{F}^{2} \\
& =\left(c_{1}-1\right)^{2}\left\|\Lambda_{\boldsymbol{\alpha} \cup \boldsymbol{\beta}_{1}^{\prime}}(X)\right\|_{F}^{2}+\left\|\Lambda_{\left(\boldsymbol{\beta} \backslash \boldsymbol{\beta}_{1}^{\prime}\right) \cup \boldsymbol{\gamma}}(X)\right\|_{F}^{2} \\
& =\left(c_{1}-1\right)^{2}\left\|\lambda_{\boldsymbol{\alpha} \cup \boldsymbol{\beta}_{1}^{\prime}}(X)\right\|_{2}^{2}+\left\|\lambda_{\left(\boldsymbol{\beta} \backslash \boldsymbol{\beta}_{1}^{\prime}\right) \cup \boldsymbol{\gamma}}(X)\right\|_{2}^{2} \\
\stackrel{(18),(8)}{=} & \left(c_{1}-1\right)^{2} c_{F}+[|\boldsymbol{\beta}|-(r-|\boldsymbol{\alpha}|)]\left(\lambda_{r}^{\mathrm{abs}}(X)\right)^{2}+\left\|\lambda_{\boldsymbol{\gamma}}(X)\right\|_{F}^{2} .
\end{aligned}
$$

Then, for any $X^{*} \in \Pi_{\mathcal{C}_{1}} \Pi_{\mathbb{S}^{n}(r)}(X)$, it takes the form in (17), namely,

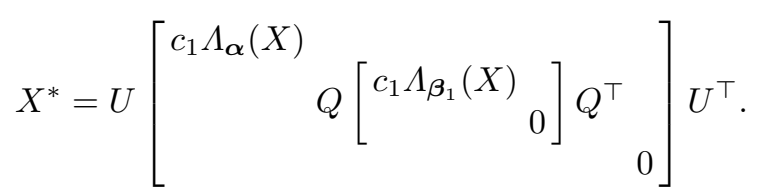

In addition,

$$
\Lambda_{\boldsymbol{\beta}}(X) \stackrel{(11)}{=} Q \Lambda_{\boldsymbol{\beta}}(X) Q^{\top}=Q\left[\begin{array}{ll}
\Lambda_{\boldsymbol{\beta}_{1}}(X) & \\
& \Lambda_{\boldsymbol{\beta} \backslash \boldsymbol{\beta}_{1}}(X)
\end{array}\right] Q^{\top},
$$

resulting in

$$
X=U\left[\begin{array}{ccc}
\Lambda_{\boldsymbol{\alpha}}(X) & & \\
& Q\left[\begin{array}{ll}
\Lambda_{\boldsymbol{\beta}_{1}}(X) & \\
& \Lambda_{\boldsymbol{\beta} \backslash \boldsymbol{\beta}_{1}}(X)
\end{array}\right] Q^{\top} & \\
& & \Lambda_{\boldsymbol{\gamma}}(X)
\end{array}\right] U^{\top} .
$$

Now, above facts allow us to derive the following chain of equations

$$
\begin{aligned}
\left\|X^{*}-X\right\|_{F}^{2} & =\left(c_{1}-1\right)^{2}\left\|\Lambda_{\boldsymbol{\alpha} \cup \boldsymbol{\beta}_{1}}(X)\right\|_{F}^{2}+\left\|\Lambda_{\left(\boldsymbol{\beta} \backslash \boldsymbol{\beta}_{1}\right) \cup \boldsymbol{\gamma}}(X)\right\|_{F}^{2} \\
& =\left(c_{1}-1\right)^{2}\left\|\lambda_{\boldsymbol{\alpha} \cup \boldsymbol{\beta}_{1}}(X)\right\|_{2}^{2}+\left\|\lambda_{\left(\boldsymbol{\beta} \backslash \boldsymbol{\beta}_{1}\right) \cup \boldsymbol{\gamma}}(X)\right\|_{2}^{2} \\
\stackrel{(18),(8)}{=} & \left(c_{1}-1\right)^{2} c_{F}+[|\boldsymbol{\beta}|-(r-|\boldsymbol{\alpha}|)]\left(\lambda_{r}^{\text {abs }}(X)\right)^{2}+\left\|\lambda_{\boldsymbol{\gamma}}(X)\right\|_{F}^{2} \\
= & f^{*} .
\end{aligned}
$$

Furthermore, clearly, $X^{*} \in \mathbb{S}^{n}(r) \cap \mathcal{C}_{1}$. Therefore, $X^{*} \in \Pi_{\mathbb{S}^{n}(r) \cap \mathcal{C}_{1}}(X)$, which implies $\Pi_{\mathbb{S}^{n}(r) \cap \mathcal{C}_{1}}(X) \supseteq \Pi_{\mathcal{C}_{1}} \Pi_{\mathbb{S}^{n}(r)}(X)$. 
Theorem 3.2 Let $X \in \mathbb{S}^{n}$ have the same EVD as in (12) and let $V_{Q}$ be given as in (9). For a set $T$, by defining $\Xi_{\mathcal{C}_{2}}^{T}(X): \mathbb{S}^{n} \rightarrow \mathbb{R}^{n}$ as

$$
\left[\Xi_{\mathcal{C}_{2}}^{T}(X)\right]_{i}=\left\{\begin{array}{cc}
\operatorname{sign}\left(\lambda_{i}(X)\right) \min \left\{\left|\lambda_{i}(X)\right|, t\right\}, & i \in T, \\
0, & \text { otherwise }
\end{array}\right.
$$

where $\operatorname{sign}(x)=1$ if $x>0, \operatorname{sign}(x)=-1$ if $x<0$, and $\operatorname{sign}(x)=0$ otherwise, it follows that

$$
\begin{aligned}
& \boldsymbol{\Pi}_{\mathbb{S}^{n}(r) \cap \mathcal{C}_{2}}(X)=\Pi_{\mathcal{C}_{2}} \boldsymbol{\Pi}_{\mathbb{S}^{n}(r)}(X) \\
= & \left\{V_{Q} \operatorname{Diag}\left(\Xi_{\mathcal{C}_{2}}^{\boldsymbol{\alpha} \cup \boldsymbol{\beta}_{2}}(X)\right) V_{Q}^{\top}: \begin{array}{l}
\text { any } Q \text { satisfies }(10), \\
\text { any } \boldsymbol{\beta}_{2} \subseteq \boldsymbol{\beta},\left|\boldsymbol{\beta}_{2}\right|=r-|\boldsymbol{\alpha}|
\end{array}\right\} .
\end{aligned}
$$

Proof. We first prove the second equation (23). For any $Q$ satisfying (10) and any $\boldsymbol{\beta}_{2} \subseteq \boldsymbol{\beta},\left|\boldsymbol{\beta}_{2}\right|=r-|\boldsymbol{\alpha}|$, every element $\Pi_{\mathbb{S}^{n}(r)}(X)$ in $\boldsymbol{\Pi}_{\mathbb{S}^{n}(r)}(X)$ from (13) takes the form

$$
\Pi_{\mathbb{S}^{n}(r)}(X)=V_{Q} \operatorname{Diag}\left(\left[\lambda_{\boldsymbol{\alpha} \cup \boldsymbol{\beta}_{2}}(X)^{\top} 0\right]^{\top}\right) V_{Q}^{\top} .
$$

Since $V_{Q} \in \mathcal{O}^{n}$, Lemma 3.1 with $\lambda^{-1}(\mathcal{K})=\mathcal{C}_{2}$ and $X=\Pi_{\mathbb{S}^{n}(r)}(X)$ indicates

$$
\Pi_{\mathcal{C}_{2}}\left(\Pi_{\mathbb{S}^{n}(r)}(X)\right)=V_{Q} \operatorname{Diag}\left(x^{*}\right) V_{Q}^{\top},
$$

where

$$
x^{*}=\operatorname{argmin}_{\|z\|_{\infty} \leq t}\left\|z-\left[\lambda_{\boldsymbol{\alpha} \cup \boldsymbol{\beta}_{2}}(X)^{\top} 0\right]^{\top}\right\|_{2}=\Xi_{\mathcal{C}_{2}}^{\boldsymbol{\alpha} \cup \boldsymbol{\beta}_{2}}(X),
$$

yielding (23). The proof of (22) is similar to that of Theorem 3.1 and is thus omitted here.

Note that $\Pi_{\mathbb{S}^{n}(r)}(X)$ keeps the $r$-largest absolute eigenvalues of $X$. Now, we consider $\Pi_{\mathbb{S}^{n}(r) \cap \mathcal{C}_{3}}(X)$. Let $\Pi_{\mathcal{L}_{r}}(X)$ be the operator that keeps the $r$-largest (not in absolute terms) eigenvalues of $X$ and sets the remaining $n-s$ to zero, and let $\Pi_{\mathcal{L}_{r}}(X)$ be the set covering all $\Pi_{\mathcal{L}_{r}}(X)$. To derive $\Pi_{\mathcal{L}_{r}}(X)$, we need the following notation similar to (8)-(10).

$$
\begin{aligned}
\boldsymbol{a} & :=\left\{i: \lambda_{i}(X)>\lambda_{r}(X)\right\}, \\
\boldsymbol{b} & :=\left\{i: \lambda_{i}(X)=\lambda_{r}(X)\right\}, \\
\boldsymbol{c} & :=\left\{i: \lambda_{i}(X)<\lambda_{r}(X)\right\}, \\
W_{P} & :=\left[\begin{array}{lll}
U_{\boldsymbol{a}} & U_{\boldsymbol{b}} P & U_{\boldsymbol{c}}
\end{array}\right], \text { where } P \in \mathcal{O}^{|\boldsymbol{b}|} .
\end{aligned}
$$

It is easy to check that

$$
W_{P} \in \mathcal{O}^{n}, \quad \Lambda_{\boldsymbol{b}}(X)=P \Lambda_{\boldsymbol{b}}(X) P^{\top} .
$$

Similarly, we now rewrite the EVD (6) as

$$
X=\left[\begin{array}{lll}
U_{a} & U_{b} & U_{c}
\end{array}\right]\left[\begin{array}{ccc}
\Lambda_{a}(X) & & \\
& \Lambda_{b}(X) & \\
& & \Lambda_{c}(X)
\end{array}\right]\left[\begin{array}{lll}
U_{a} & U_{b} & U_{c}
\end{array}\right]^{\top}
$$


and then $\boldsymbol{\Pi}_{\mathcal{L}_{r}}(X)$ is given by

$$
\boldsymbol{\Pi}_{\mathcal{L}_{r}}(X)=\left\{W_{P} \operatorname{Diag}(y) W_{P}^{\top}: \text { any } y \in \mathcal{Y} \text { and any } P \in \mathcal{O}^{|\boldsymbol{b}|}\right\}
$$

where

$$
\mathcal{Y}=\left\{y \in \mathbb{R}^{n}: y_{i}=\left\{\begin{array}{cc}
\lambda_{i}(X), & i \in \boldsymbol{a} \cup \boldsymbol{b}_{0} \\
0, & \text { otherwise }
\end{array} \text { with any } \boldsymbol{b}_{0} \subseteq \boldsymbol{b},\left|\boldsymbol{b}_{0}\right|=r-|\boldsymbol{a}|\right\} .\right.
$$

Theorem 3.3 Let $X \in \mathbb{S}^{n}$ have the same EVD as in (29) and let $W_{\mathrm{P}}$ be given as in (27). For a set $T$, by defining $\Xi_{\mathcal{C}_{3}}^{T}(X): \mathbb{S}^{n} \rightarrow \mathbb{R}^{n}$ as

$$
\left[\Xi_{\mathcal{C}_{3}}^{T}(X)\right]_{i}=\left\{\begin{array}{cl}
\max \left\{\lambda_{i}(X)-\frac{1}{\varrho}\left(\sum_{j=1}^{\varrho} \lambda_{j}(X)-1\right), 0\right\} & i \in T \\
0, & \text { otherwise }
\end{array}\right.
$$

where $\varrho:=\max \left\{j: \lambda_{j}(X)>\frac{1}{j}\left(\sum_{i=1}^{j} \lambda_{i}(X)-1\right)\right\}$, it follows that

$$
\begin{aligned}
& \Pi_{\mathbb{S}^{n}(r) \cap \mathcal{C}_{3}}(X)=\Pi_{\mathcal{C}_{3}} \Pi_{\mathcal{L}_{r}}(X) \\
& =\left\{W_{P} \operatorname{Diag}\left(\Xi_{\mathcal{C}_{3}}^{\boldsymbol{a} \cup \boldsymbol{b}_{3}}(X)\right) W_{P}^{\top}: \begin{array}{l}
\text { any } P \in \mathcal{O}^{|\boldsymbol{b}|} \\
\text { any } \boldsymbol{b}_{3} \subseteq \boldsymbol{b},\left|\boldsymbol{b}_{3}\right|=r-|\boldsymbol{a}|
\end{array}\right\} \text {. }
\end{aligned}
$$

Proof. We first prove the second equation (33). For any $P \in \mathcal{O}^{|\boldsymbol{b}|}$ and any $\boldsymbol{b}_{3} \subseteq \boldsymbol{b},\left|\boldsymbol{b}_{3}\right|=r-|\boldsymbol{a}|$, every element $\Pi_{\mathcal{L}_{r}}(X)$ in $\boldsymbol{\Pi}_{\mathcal{L}_{r}}(X)$ from (30) satisfies

$$
\Pi_{\mathcal{L}_{r}}(X)=W_{P} \operatorname{Diag}\left(\left[\lambda_{\boldsymbol{a} \cup \boldsymbol{b}_{3}}(X)^{\top} 0\right]^{\top}\right) W_{P}^{\top} .
$$

Since $W_{P} \in \mathcal{O}^{n}$, Lemma 3.1 with $\lambda^{-1}(\mathcal{K})=\mathcal{C}_{3}$ and $X=\Pi_{\mathcal{L}^{n}(r)}(X)$ indicates

$$
\Pi_{\mathcal{C}_{3}}\left(\Pi_{\mathcal{L}_{r}}(X)\right)=W_{P} \operatorname{Diag}\left(x^{*}\right) W_{P}^{\top},
$$

where

$$
x^{*}=\operatorname{argmin}_{z \in \mathcal{K}_{3}}\left\|z-\left[\lambda_{\boldsymbol{a} \cup \boldsymbol{b}_{3}}(X)^{\top} 0\right]^{\top}\right\|_{2}=\Xi_{\mathcal{C}_{3}}^{\boldsymbol{a} \cup \boldsymbol{b}_{3}}(X),
$$

where the last equality follows [41, Lemma 3](Page 16), which verifies (33). Next, we prove (32) through proving two parts. In the first part, we prove that $\Pi_{\mathbb{S}^{n}(r) \cap \mathcal{C}_{3}}(X) \subseteq \Pi_{\mathcal{C}_{3}} \Pi_{\mathcal{L}_{r}}(X)$. In the second part, we show the reverse inclusion.

Part 1. For any $\Pi_{\mathbb{S}^{n}}(r) \cap \mathcal{C}_{3}(X) \in \Pi_{\mathbb{S}^{n}(r) \cap \mathcal{C}_{3}}(X)$, Lemma 3.1, in which $\lambda^{-1}(\mathcal{K})=\mathbb{S}^{n}(r) \cap \mathcal{C}_{3}$, indicates

$$
\Pi_{\mathbb{S}^{n}(r) \cap \mathcal{C}_{3}}(X)=\bar{U} \operatorname{Diag}\left(x^{*}\right) \bar{U}^{\top},
$$

where $\bar{U}=\left[\begin{array}{lll}U_{\boldsymbol{a}} & U_{\boldsymbol{b}} & U_{\boldsymbol{c}}\end{array}\right]$ and $x^{*}$ is an optimal solution of

$$
\min \left\{\|z-\lambda(X)\|_{2}: z \in \mathcal{K}_{3} \cap \mathbb{R}^{n}(r)\right\} .
$$

The above problem is equivalent to

$$
\min _{z}\left\{\begin{array}{ll}
\left\|z_{a \cup b_{3}}-\lambda_{a \cup b_{3}}(X)\right\|_{2}: \begin{array}{l}
\left\|z_{\boldsymbol{a} \cup \boldsymbol{b}_{3}}\right\|_{1}=1, z_{\boldsymbol{a} \cup \boldsymbol{b}_{3} \geq 0} \geq 0 \\
z_{i}=0, i \notin \boldsymbol{a} \cup \boldsymbol{b}_{3}
\end{array}
\end{array}\right\}
$$


for any $\boldsymbol{b}_{3} \subseteq \boldsymbol{b},\left|\boldsymbol{b}_{3}\right|=r-|\boldsymbol{a}|$ owing to [41, Algorithm 1] (Page 17), where $\boldsymbol{a}, \boldsymbol{b}$ are defined as in (24-25). The rest of the proof of Part 1 to verify

$$
\Pi_{\mathbb{S}^{n}(r) \cap \mathcal{C}_{3}}(X) \subseteq \Pi_{\mathcal{C}_{3}} \Pi_{\mathcal{L}_{r}}(X)
$$

is similar to that of Part 1 in Theorem 3.1.

Part 2. The idea is the same as that used to prove Part $\mathbf{2}$ in Theorem 3.1. From the proof in Part 1, we can see that for a given $\boldsymbol{b}_{3}^{\prime} \subseteq \boldsymbol{b},\left|\boldsymbol{b}_{3}^{\prime}\right|=r-|\boldsymbol{a}|$,

$$
Y^{*}:=\bar{U}\left[\begin{array}{ll}
\operatorname{Diag}\left(\left(\bar{\lambda}_{i}(X)\right)_{i \in \boldsymbol{a} \cup \boldsymbol{b}_{3}^{\prime}}\right) & \\
& 0
\end{array}\right] \bar{U}^{\top} \in \Pi_{\mathcal{C}_{3}} \boldsymbol{\Pi}_{\mathcal{L}_{r}}(X)
$$

is also $Y^{*} \in \boldsymbol{\Pi}_{\mathbb{S}^{n}(r) \cap \mathcal{C}_{3}}(X)$, where $\bar{U}=\left[\begin{array}{lll}U_{\boldsymbol{a}} & U_{\boldsymbol{b}} & U_{\boldsymbol{c}}\end{array}\right]$ and

$$
\bar{\lambda}_{i}(X):=\max \left\{\lambda_{i}(X)-\frac{1}{\varrho}\left(\sum_{j=1}^{\varrho} \lambda_{j}(X)-1\right), 0\right\}, \quad i \in \boldsymbol{a} \cup \boldsymbol{b}_{3}^{\prime} .
$$

In addition,

$$
X \stackrel{(29)}{=} \bar{U}\left[\begin{array}{lll}
\Lambda_{\boldsymbol{a}}(X) & & \\
& \Lambda_{\boldsymbol{b}}(X) & \\
& & \Lambda_{\boldsymbol{c}}(X)
\end{array}\right] \bar{U}^{\top} .
$$

Therefore, the optimal objective function value is

$$
\begin{aligned}
f^{*} & =\min _{Y \in \mathbb{S}^{n}(r) \cap \mathcal{C}_{3}}\|Y-X\|_{F}^{2}=\left\|Y^{*}-X\right\|_{F}^{2} \\
& =\left\|\bar{\lambda}_{\boldsymbol{a}}(X)-\lambda_{\boldsymbol{a}}(X)\right\|_{2}^{2}+\left\|\bar{\lambda}_{\boldsymbol{b}_{3}^{\prime}}(X)-\lambda_{\boldsymbol{b}_{3}^{\prime}}(X)\right\|_{2}^{2}+\left\|\lambda_{\boldsymbol{b} \backslash \boldsymbol{b}_{3}^{\prime}}(X)\right\|_{2}^{2}+\left\|\lambda_{\boldsymbol{c}}(X)\right\|_{2}^{2} .
\end{aligned}
$$

Then, from (33), any $X^{*} \in \Pi_{\mathcal{C}_{3}} \boldsymbol{\Pi}_{\mathcal{L}_{r}}(X)$ can be expressed as

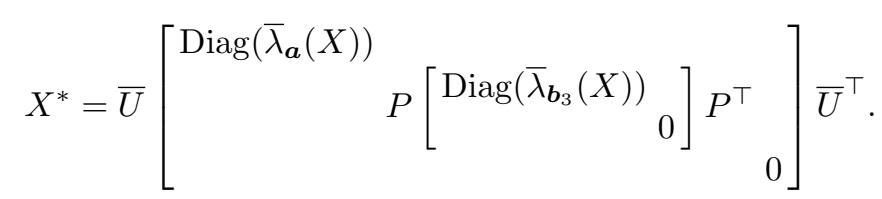

Moreover,

$$
\Lambda_{\boldsymbol{b}}(X) \stackrel{(28)}{=} P \Lambda_{\boldsymbol{b}}(X) P^{\top}=P\left[\begin{array}{ll}
\Lambda_{\boldsymbol{b}_{3}}(X) & \\
& \Lambda_{\boldsymbol{b} \backslash \boldsymbol{b}_{3}}(X)
\end{array}\right] P^{\top},
$$

resulting in

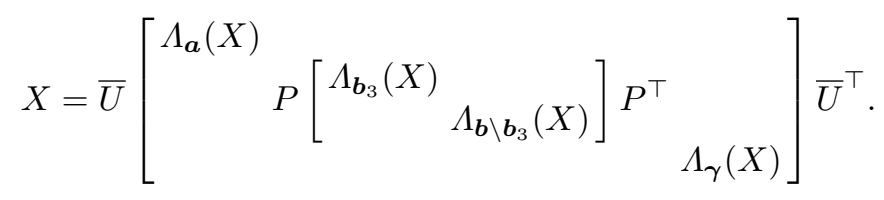


These facts suffice to show that

$$
\begin{aligned}
& \left\|X^{*}-X\right\|_{F}^{2} \\
= & \left\|\bar{\lambda}_{\boldsymbol{a}}(X)-\lambda_{\boldsymbol{a}}(X)\right\|_{2}^{2}+\left\|\bar{\lambda}_{\boldsymbol{b}_{3}}(X)-\lambda_{\boldsymbol{b}_{3}}(X)\right\|_{2}^{2}+\left\|\lambda_{\boldsymbol{b} \backslash \boldsymbol{b}_{3}}(X)\right\|_{2}^{2}+\left\|\lambda_{\boldsymbol{c}}(X)\right\|_{2}^{2} \\
= & \left\|\bar{\lambda}_{\boldsymbol{a}}(X)-\lambda_{\boldsymbol{a}}(X)\right\|_{2}^{2}+\left\|\bar{\lambda}_{\boldsymbol{b}_{3}^{\prime}}(X)-\lambda_{\boldsymbol{b}_{3}^{\prime}}(X)\right\|_{2}^{2}+\left\|\lambda_{\boldsymbol{b} \backslash \boldsymbol{b}_{3}^{\prime}}(X)\right\|_{2}^{2}+\left\|\lambda_{\boldsymbol{c}}(X)\right\|_{2}^{2} \\
= & f^{*},
\end{aligned}
$$

where the second equality is based on three facts: (i) $\boldsymbol{b}_{3} \subseteq \boldsymbol{b}, \boldsymbol{b}_{3}^{\prime} \subseteq \boldsymbol{b}$, (ii) $\lambda_{i}(X)=\lambda_{r}(X)$, and (iii) $\bar{\lambda}_{i}(X)=\max \left\{\lambda_{r}(X)-\frac{1}{\varrho}\left(\sum_{j=1}^{\varrho} \lambda_{j}(X)-1\right), 0\right\}$ for any $i \in \boldsymbol{b}$. Moreover, $X^{*} \in \mathbb{S}^{n}(r) \cap \mathcal{C}_{3}$. Therefore, $X^{*} \in \boldsymbol{\Pi}_{\mathbb{S}^{n}}(r) \cap \mathcal{C}_{3}(X)$, which implies $\Pi_{\mathbb{S}^{n}(r) \cap \mathcal{C}_{3}}(X) \supseteq \Pi_{\mathcal{C}_{3}} \Pi_{\mathcal{L}_{r}}(X)$.

\section{Normal Cones}

In this section, we provide a decomposition property of the Fréchet normal cone to $\mathbb{S}^{n}(r) \cap \mathcal{C}$ to prepare for the optimality conditions of problem (1) in Section 5. Let $\Omega$ be a closed and non-empty subset in $\mathbb{S}^{n}$ and $X \in \Omega$. A matrix $Z$ is Fréchet normal (also called prenormal in [42]) to $\Omega$ at $X$ provided that for every $\varepsilon>0$, there exists a $\theta>0$ such that

$$
\langle Z, Y-X\rangle \leq \varepsilon\|Y-X\|_{F}, \quad \text { for all } Y \in(X+\theta \mathbf{B}) \cap \Omega,
$$

where $\mathbf{B}$ is a unit ball in $\mathbb{S}^{n}$ and $\langle X, Y\rangle=\operatorname{Tr}(X Y)$. Fréchet normal cone $\mathrm{N}_{\Omega}^{F}(X)$ (also written as $\widehat{\mathrm{N}}_{\Omega}(X)$ in [26]) is the set containing all these matrices $Z$. For any closed and convex set $\Omega$ and any $X \in \Omega$, one has

$$
\mathrm{N}_{\Omega}^{F}(X)=\mathrm{N}_{\Omega}^{\text {conv }}(X),
$$

where $\mathrm{N}_{\Omega}^{\text {conv }}(X)$ is the convex normal cone in convex analysis, namely,

$$
\mathrm{N}_{\Omega}^{\text {conv }}(X)=\left\{W \in \mathbb{S}^{n}:\langle W, Y-X\rangle \leq 0, \forall Y \in \Omega\right\} .
$$

The indicator function of set $\Omega$ is given as $\delta_{\Omega}(x)=0$ if $x \in \Omega$ and $\delta_{\Omega}(x)=+\infty$ otherwise. Let $\operatorname{bd} \Omega$ and $\operatorname{int} \Omega$ stand for the boundary and interior of set $\Omega$, respectively.

A closed formula for the Fréchet normal cone to the set of low-rank (possibly rectangular) real matrices has been derived by $[27,28]$. This formula can be directly applied to the space of a symmetric matrix.

Lemma 4.1 For $X \in \mathbb{S}^{n}(r)$ with the same $E V D$ as in (6) and $s:=\operatorname{rank}(X)$, the Fréchet normal cone to $\mathbb{S}^{n}(r)$ at $X$ is given by

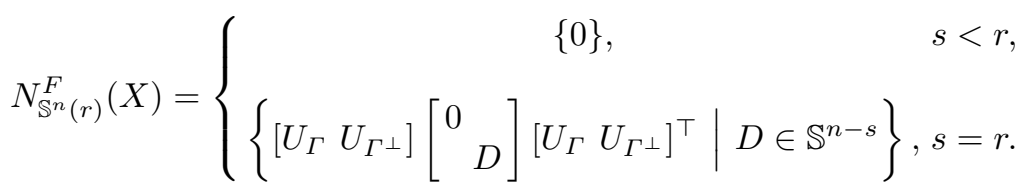


The next results present the normal cones to sets $\mathcal{C}$. We would like to define some notation here. For $X \in \mathcal{C}$ with the EVD in (6) and letting $s:=\operatorname{rank}(X)$, we define two types of matrices. For $Y \in \mathbb{S}^{s}, Z \in \mathbb{S}^{n-s}$, define

$$
\mathcal{M}_{X}(Y, Z):=\left[\begin{array}{ll}
U_{\Gamma} & U_{\Gamma^{\perp}}
\end{array}\right]\left[\begin{array}{ll}
Y & \\
& Z
\end{array}\right]\left[\begin{array}{ll}
U_{\Gamma} & U_{\Gamma^{\perp}}
\end{array}\right]^{\top} .
$$

Particularly, for $\mathcal{C}_{2}$, let

$$
\begin{array}{ll}
\boldsymbol{\eta}_{+}:=\left\{i: \lambda_{i}(X)=t\right\}, & \boldsymbol{\eta}_{-}:=\left\{i: \lambda_{i}(X)=-t\right\} \\
\boldsymbol{\eta}_{ \pm}:=\left\{i: 0<\left|\lambda_{i}(X)\right|<t\right\}, & \boldsymbol{\eta}_{=}:=\left\{i: \lambda_{i}(X)=0\right\}
\end{array}
$$

For $A \in \mathbb{S}^{\left|\boldsymbol{\eta}_{+}\right|}, B \in \mathbb{S}^{\left|\boldsymbol{\eta}_{ \pm}\right|}, C \in \mathbb{S}^{\left|\boldsymbol{\eta}_{-}\right|}, D \in \mathbb{S}^{\left|\boldsymbol{\eta}_{=}\right|}$, define

$$
\begin{gathered}
\mathcal{N}_{X}(A, B, C, D) \\
:=\left[U_{\boldsymbol{\eta}_{+}} U_{\boldsymbol{\eta}_{ \pm}} U_{\boldsymbol{\eta}_{-}} U_{\boldsymbol{\eta}_{=}}\right]\left[\begin{array}{cccc}
A & & & \\
& B & & \\
& & C & \\
& & & D
\end{array}\right]\left[U_{\boldsymbol{\eta}_{+}} U_{\boldsymbol{\eta}_{ \pm}} U_{\boldsymbol{\eta}_{-}} U_{\boldsymbol{\eta}_{=}}\right]^{\top} .
\end{gathered}
$$

Clearly, one can check that if $X \in \mathcal{C}_{2}$, then

$$
\Gamma=\boldsymbol{\eta}_{+} \cup \boldsymbol{\eta}_{ \pm} \cup \boldsymbol{\eta}_{-}, \quad \Gamma^{\perp}=\boldsymbol{\eta}_{=} .
$$

As a closed and convex set, each $\mathcal{K}_{i}$ usually appears in the vector space. Its normal cone has been given in [43, Examples 5.14 and 5.2.6] and [26, Example 6.10] and allows us to obtain the normal cone of $\mathcal{C}_{i}$ by the following lemma.

Lemma $4.2\left[39\right.$, Fact 2.4] Let $\mathcal{K} \in \mathbb{R}^{n}$ be a closed symmetric set. For any $X \in \lambda^{-1}(\mathcal{K})$, the Fréchet normal cone to the spectral set $\lambda^{-1}(\mathcal{K})$ is given by

$$
\mathrm{N}_{\lambda^{-1}(\mathcal{K})}^{F}(X)=\left\{U \operatorname{Diag}(y) U^{\top}: y \in \mathrm{N}_{\mathcal{K}}^{F}(\lambda(X)), U \in \mathcal{O}^{n}(X)\right\} .
$$

Proof. Since $\mathcal{K}$ is closed and symmetric, its indicator function $\delta_{\mathcal{K}}$ is lower semicontinuous and symmetric. Then, the result directly follows from [44, Theorem 4.2] in the sense of the Fréchet normal.

Proposition 4.1 (Normal cones to $\mathcal{C}$ ) For $X \in \mathcal{C}$ with the EVD in (6) and $s:=\operatorname{rank}(X)=|\Gamma|$, the normal cone to $\mathcal{C}$ at $X$ is given as follows.

(i) If $X \in$ int $\mathcal{C}_{1}$, then $\mathrm{N}_{\mathcal{C}_{1}}^{F}(X)=\{0\}$. If $X \in \operatorname{bd} \mathcal{C}_{1}$, then

$$
\mathrm{N}_{\mathcal{C}_{1}}^{F}(X)=\left\{\mathcal{M}_{X}\left(\rho \Lambda_{\Gamma}(X), 0\right): \rho \geq 0\right\} .
$$

(ii) If $X \in \operatorname{int} \mathcal{C}_{2}$, then $\mathrm{N}_{\mathcal{C}_{2}}^{F}(X)=\{0\}$. If $X \in \mathrm{bd} \mathcal{C}_{2}$, then

$$
\mathrm{N}_{\mathcal{C}_{2}}^{F}(X)=\left\{\mathcal{N}_{X}(A, 0, C, 0): A \in \mathbb{S}_{+}^{\left|\boldsymbol{\eta}_{+}\right|}, C \in \mathbb{S}_{-}^{\left|\boldsymbol{\eta}_{-}\right|}\right\} .
$$


(iii) If $X \in \mathcal{C}_{3}$, then

$$
\mathrm{N}_{\mathcal{C}_{3}}^{F}(X)=\left\{\mathcal{M}_{X}\left(\rho I_{s}, \rho I_{n-s}+D\right): \rho \in \mathbb{R}, D \in \mathbb{S}_{-}^{n-s}\right\} .
$$

Proof. (i) Let $X \in \mathcal{C}_{1}$. Then, from [43, Examples 5.14] and Lemma 4.2, the normal cone to $\mathcal{C}_{1}$ at $X$ is given by

$$
\mathrm{N}_{\mathcal{C}_{1}}^{F}(X)=\left\{\begin{array}{c}
\{0\}, \quad \text { for } X \in \operatorname{int} \mathcal{C}_{1}, \\
\rho X, \text { with } \rho \geq 0, \text { for } X \in \operatorname{bdC}_{1} .
\end{array}\right.
$$

This result, together with the EVD in (6), yields the conclusion immediately.

(ii) Let $X \in \mathcal{C}_{2}$. Clearly, $\mathrm{N}_{\mathcal{C}_{2}}^{\text {conv }}(X)=\{0\}$ when $X \in \operatorname{int} \mathcal{C}_{2}$. Consider the case of $X \in \operatorname{bd} \mathcal{C}_{2}$. Decompose

$$
\mathcal{C}_{2}=\mathcal{C}_{2}^{1} \times \mathcal{C}_{2}^{2} \times \cdots \times \mathcal{C}_{2}^{n},
$$

where each $\mathcal{C}_{2}^{i}:=\left\{X \in \mathbb{S}^{n}:-t \leq \lambda_{i}(X) \leq t\right\}$ is a closed interval. Then, by [26, Example 6.10] and Lemma 4.2, the normal cone to $\mathcal{C}_{2}$ at $X$ takes the form

$$
\mathrm{N}_{\mathcal{C}_{2}}^{F}(X)=\mathrm{N}_{\mathcal{C}_{2}^{1}}^{F}\left(\lambda_{1}(X)\right) \times \mathrm{N}_{\mathcal{C}_{2}^{2}}^{F}\left(\lambda_{2}(X)\right) \times \cdots \times \mathrm{N}_{\mathcal{C}_{2}^{n}}^{F}\left(\lambda_{n}(X)\right),
$$

where

$$
\mathrm{N}_{\mathcal{C}_{2}^{i}}^{F}\left(\lambda_{i}(X)\right)=\left\{c u_{i} u_{i}^{\top}: c\left\{\begin{array}{l}
\geq 0, i \in \boldsymbol{\eta}_{+} \\
\leq 0, i \in \boldsymbol{\eta}_{-} \\
=0, \text { otherwise }
\end{array}\right\} .\right.
$$

This result derives the conclusion immediately.

(iii) Let $X \in \mathcal{C}_{3}$. Clearly, $\mathcal{C}_{3}$ is the intersection of $\mathbb{S}_{+}^{n}$ and

$$
\Delta:=\left\{X \in \mathbb{S}^{n} \mid \operatorname{Tr}(X)=1\right\} .
$$

Then, by [43, Proposition 5.3.1 and Remark 5.3.2], the normal cone to $\mathcal{C}_{3}$ at $X$ is given by

$$
\begin{aligned}
\mathrm{N}_{\mathcal{C}_{3}}^{F}(X) & =\mathrm{N}_{\Delta}^{F}(X)+\mathrm{N}_{\mathbb{S}_{+}^{n}}^{F}(X) \\
& =\left\{\rho I_{n}: \rho \in \mathbb{R}\right\}+\left\{W \in \mathbb{S}^{n}: \operatorname{tr}(X W)=0, W \in \mathbb{S}_{-}^{n}\right\} .
\end{aligned}
$$

Since $X$ has the same EVD as in (6), then

$$
\mathrm{N}_{\mathbb{S}_{+}^{n}}^{F}(X)=\left\{\mathcal{M}_{X}(0, D): D \in \mathbb{S}_{-}^{n}\right\} .
$$

This result concludes the proof.

To end this section, we calculate the normal cone to $\mathbb{S}^{n}(r) \cap \mathcal{C}$, which turns out to be a summation of the normal cone to $\mathbb{S}^{n}(r)$ and the normal cone to $\mathcal{C}$.

Theorem 4.1 Let $X \in \mathbb{S}^{n}(r) \cap \mathcal{C}$. We have

$$
\mathrm{N}_{\mathbb{S}^{n}(r) \cap \mathcal{C}}^{F}(X)=\mathrm{N}_{\mathbb{S}^{n}(r)}^{F}(X)+\mathrm{N}_{\mathcal{C}}^{F}(X) .
$$


Proof. Since $\mathbb{R}^{n}(r) \cap \mathcal{K}$ is closed and symmetric and it is easy to verify that $\mathbb{S}^{n}(r) \cap \mathcal{C}=\lambda^{-1}\left(\mathbb{R}^{n}(r) \cap \mathcal{K}\right)$, we have the following chain of equations

$$
\begin{aligned}
& \mathrm{N}_{\mathbb{S}^{n}(r) \cap \mathcal{C}}^{F}(X) \\
= & \mathrm{N}_{\lambda^{-1}\left(\mathbb{R}^{n}(r) \cap \mathcal{K}\right)}^{F}(X) \\
\text { Lemma4.2 }^{\prime} & \left\{U \operatorname{Diag}(w) U^{\top}: w \in \mathrm{N}_{\mathbb{R}^{n}(r) \cap \mathcal{K}}^{F}(\lambda(X)), U \in \mathcal{O}^{n}(X)\right\} \\
= & \left\{U \operatorname{Diag}(w) U^{\top}: w \in \mathrm{N}_{\mathbb{R}^{n}(r)}^{F}(\lambda(X))+N_{\mathcal{K}}^{F}(\lambda(X)), U \in \mathcal{O}^{n}(X)\right\} \\
= & \left\{U \operatorname{Diag}(w) U^{\top}: w \in \mathrm{N}_{\mathbb{R}^{n}(r)}^{F}(\lambda(X)), U \in \mathcal{O}^{n}(X)\right\} \\
+ & \left\{U \operatorname{Diag}(w) U^{\top}: w \in \mathrm{N}_{\mathcal{K}}^{F}(\lambda(X)), U \in \mathcal{O}^{n}(X)\right\} \\
\text { Lemma4.2 } & \mathrm{N}_{\lambda^{-1}\left(\mathbb{R}^{n}(r)\right)}^{F}(X)+\mathrm{N}_{\lambda^{-1}(\mathcal{K})}^{F}(X) \\
= & \mathrm{N}_{\mathbb{S}^{n}(r)}^{F}(X)+\mathrm{N}_{\mathcal{C}}^{F}(X),
\end{aligned}
$$

where the third equation holds from $\mathrm{N}_{\mathbb{R}^{n}(r) \cap \mathcal{K}}^{F}(x)=\mathrm{N}_{\mathbb{R}^{n}(r)}^{F}(x)+\mathrm{N}_{\mathcal{K}}^{F}(x)$ for $x \in \mathbb{R}^{n}(r) \cap \mathcal{K}$ by [45, Corollary 4.1] or [46, Corollary 2.9] for $\mathcal{K}=\mathcal{K}_{1}, \mathcal{K}_{2}, \mathcal{K}_{3}$. The proof is thus complete.

Remark 4.1 It is worth mentioning that (41) may not hold for other general sets $\mathcal{C}$. To derive it, some basic qualification conditions [26, Theorem 6.42] are necessary. However, these qualification conditions are naturally satisfied when $\mathcal{C}$ is one of $\mathcal{C}_{1}, \mathcal{C}_{2}$ and $\mathcal{C}_{3}$. Therefore, (41) allows us to calculate $\mathrm{N}_{\mathbb{S}^{n}(r) \cap \mathcal{C}}^{F}(X)$ easily through Lemma 4.1 and Proposition 4.1.

\section{Stationary Points}

In this section, we investigate the first-order optimality conditions of problem (1), starting with the definitions of two types of stationary points. The $\alpha$ stationary point defined below is actually a generalization of the concept of the $L$-stationary point for the sparsity constrained optimization of Beck and Eldar in [47, Definition 2.3].

Definition 5.1 Let $X \in \mathbb{S}^{n}(r) \cap \mathcal{C}$ be a given feasible point of (1).

(i) We say $X$ is an $F$-stationary point of (1) if

$$
0 \in \nabla f(X)+N_{\mathbb{S}^{n}(r)}^{F}(X)+N_{\mathcal{C}}^{F}(X) .
$$

(ii) We say $X$ is an $\alpha$-stationary point of (1) if, for a given $\alpha>0$,

$$
X \in \Pi_{\mathbb{S}^{n}(r) \cap \mathcal{C}}(X-\alpha \nabla f(X)) .
$$

Using the formulas of the Fréchet normal cone in Section 4 and the properties of projection onto $\mathbb{S}^{n}(r) \cap \mathcal{C}$ in Section 3, we present more explicit representations of an $F$-stationary point and an $\alpha$-stationary point. 
Theorem 5.1 For any $X \in \mathbb{S}^{n}(r) \cap \mathcal{C}_{1}$ with the same EVD as in (6), denote $s:=\operatorname{rank}(X)=|\Gamma|$ and

$$
\Omega_{\mathcal{C}_{1}}(X):= \begin{cases}\{0\}, & X \in \operatorname{int} \mathcal{C}_{1} \\ \{\rho \in \mathbb{R} \mid \rho \leq 0\}, & X \in \mathrm{bdC}_{1}\end{cases}
$$

We have the following results.

(i) $X$ is an F-stationary point of (1) if and only if

$$
\nabla f(X) \in \begin{cases}\left\{\rho X: \rho \in \Omega_{\mathcal{C}_{1}}(X)\right\}, & s<r \\
\left\{\mathcal{M}_{X}\left(\rho \Lambda_{\Gamma}(X), D\right): \begin{array}{l}
\rho \in \Omega_{\mathcal{C}_{1}}(X) \\
D \in \mathbb{S}^{n-s}
\end{array}\right\}, s=r .\end{cases}
$$

(ii) $X$ is an $\alpha$-stationary point of (1) for a given $\alpha>0$ if and only if

$$
\nabla f(X) \in\left\{\mathcal{M}_{X}\left(\rho \Lambda_{\Gamma}(X), D\right): \begin{array}{l}
\rho \in \Omega_{\mathcal{C}_{1}}(X), D \in \mathbb{S}^{n-s} \\
\text { with } \bar{\lambda}_{D} \leq\left(\frac{1}{\alpha}-\rho\right) \lambda_{r}^{\text {abs }}(X)
\end{array}\right\}
$$

where $\bar{\lambda}_{D}:=\max _{i \in\{1, \cdots, n-s\}}\left|\lambda_{i}(D)\right|$.

Proof. (i) By Lemma 4.1, Proposition 4.1 (i) and the sum rule of Theorem 4.1 , we can obtain the formula of $N_{\mathbb{S}^{n}(r) \cap \mathcal{C}_{1}}^{F}(X)$, which allows us to reach the conclusion.

(ii) Sketch of the proof: For the 'If' part, we need to show that

$$
\nabla f(X) \in \Theta_{1} \Longrightarrow X \in \Pi_{\mathbb{S}^{n}(r) \cap \mathcal{C}_{1}}(X-\alpha \nabla f(X)), \forall X \in \mathbb{S}^{n}(r) \cap \mathcal{C}_{1}
$$

where $\Theta_{1}$ is the set of the right hand side of (45). We prove this part by considering two cases: $X \in \mathrm{bd}_{1}$ and $X \in \operatorname{int} \mathcal{C}_{1}$. For the 'Only if' part, we need to show

$$
X \in \boldsymbol{\Pi}_{\mathbb{S}^{n}(r) \cap \mathcal{C}_{1}}(X-\alpha \nabla f(X)) \quad \Longrightarrow \quad \nabla f(X) \in \Theta_{1}
$$

To do this, we first prove that $X$ and $X^{\prime}:=X-\alpha \nabla f(X)$ have a decomposition similar to

$$
X=U\left[\begin{array}{ll}
\Lambda_{\Gamma}(X) & \\
& 0
\end{array}\right] U^{\top}, \quad X^{\prime}=U\left[\begin{array}{ll}
\Lambda_{1} & \\
& \Lambda_{2}
\end{array}\right] U^{\top},
$$

where $\Lambda_{1}, \Lambda_{2}$ will be specified later, which suffices to show that

$$
\nabla f(X)=\mathcal{M}_{X}\left(\rho \Lambda_{\Gamma}(X), D\right)
$$

We then show $\rho \leq 0$ and $\bar{\lambda}_{D} \leq(1 / \alpha-\rho) \lambda_{r}^{\text {abs }}(X)$ by means of the two cases of $X \in \operatorname{bd} \mathcal{C}_{1}$ and $X \in \operatorname{int} \mathcal{C}_{1}$. 
'If' part: Case 1) Suppose $X \in \mathrm{bd}_{1}$. It follows from $\nabla f(X) \in \Theta_{1}$ that

$$
\begin{aligned}
& \Pi_{\mathbb{S}^{n}(r) \cap \mathcal{C}_{1}}(X-\alpha \nabla f(X)) \\
= & \Pi_{\mathbb{S}^{n}(r) \cap \mathcal{C}_{1}}\left(\mathcal{M}_{X}\left(\Lambda_{\Gamma}(X), 0\right)-\alpha \mathcal{M}_{X}\left(\rho \Lambda_{\Gamma}(X), D\right)\right) \\
= & \Pi_{\mathbb{S}^{n}(r) \cap \mathcal{C}_{1}}\left(\mathcal{M}_{X}\left((1-\alpha \rho) \Lambda_{\Gamma}(X),-\alpha D\right)\right) \\
\stackrel{(16)}{=} & \Pi_{\mathcal{C}_{1}} \Pi_{\mathbb{S}^{n}(r)}\left(\mathcal{M}_{X}\left((1-\alpha \rho) \Lambda_{\Gamma}(X),-\alpha D\right)\right) \\
= & \Pi_{\mathcal{C}_{1}}\left(\mathcal{M}_{X}\left((1-\alpha \rho) \Lambda_{\Gamma}(X), 0\right)\right) \\
= & \Pi_{\mathcal{C}_{1}}((1-\alpha \rho) X) \\
= & X
\end{aligned}
$$

where the fourth equality holds because

$$
\begin{aligned}
\max _{i \in\{1, \cdots, n-s\}}\left|\lambda_{i}(-\alpha D)\right| & =\alpha \max _{i \in\{1, \cdots, n-s\}}\left|\lambda_{i}(D)\right| \\
& =\alpha \bar{\lambda}_{D} \leq(1-\alpha \rho) \lambda_{r}^{\mathrm{abs}}(X) \\
& = \begin{cases}0, & r<s, \\
(1-\alpha \rho) \min _{i \in \Gamma}\left|\lambda_{i}(X)\right|, & r=s,\end{cases}
\end{aligned}
$$

which implies $X \in \boldsymbol{\Pi}_{\mathbb{S}^{n}}(r) \cap \mathcal{C}_{1}(X-\alpha \nabla f(X))$. Case 2) Suppose $X \in \operatorname{int}_{1}$. This is a special case of Case 1) with $\rho=0$.

'Only if' part: For any $X \in \boldsymbol{\Pi}_{\mathbb{S}^{n}}(r) \cap \mathcal{C}_{1}(X-\alpha \nabla f(X))$, let

$$
X^{\prime}:=X-\alpha \nabla f(X)=U^{\prime} \operatorname{Diag}\left(\lambda\left(X^{\prime}\right)\right)\left(U^{\prime}\right)^{\top} .
$$

Let $\boldsymbol{\alpha}^{\prime}, \boldsymbol{\beta}^{\prime}, \boldsymbol{\gamma}^{\prime}$ be defined as in (8) and $Q^{\prime}$ be defined as in (10), in which $X$ is replaced by $X^{\prime}$. It follows from (17) that for any $X \in \boldsymbol{\Pi}_{\mathbb{S}^{n}}(r) \cap \mathcal{C}_{1}\left(X^{\prime}\right)$, there is a $Q^{\prime}$ satisfying (10) and a $\boldsymbol{\beta}_{1}^{\prime} \subseteq \boldsymbol{\beta}^{\prime},\left|\boldsymbol{\beta}_{1}^{\prime}\right|=r-\left|\boldsymbol{\alpha}^{\prime}\right|$ such that

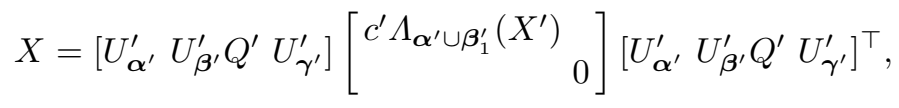

where

$$
\begin{aligned}
c^{\prime} & :=\min \left\{1,\left(\left\|\lambda_{\boldsymbol{\alpha}^{\prime}}\left(X^{\prime}\right)\right\|_{2}^{2}+\left(r-\left|\boldsymbol{\alpha}^{\prime}\right|\right)\left(\lambda_{r}^{\mathrm{abs}}\left(X^{\prime}\right)\right)^{2}\right)^{-1 / 2}\right\} \\
& =\min \{1,1 / \phi\}, \text { where } \phi:=\left\|\Lambda_{\boldsymbol{\alpha}^{\prime} \cup \boldsymbol{\beta}_{1}^{\prime}}\left(X^{\prime}\right)\right\|_{F} .
\end{aligned}
$$

Recall that $X$ has the same EVD as in (6), i.e.,

$$
X=U\left[\begin{array}{cc}
\Lambda_{\Gamma}(X) & \\
& 0
\end{array}\right] U^{\top} .
$$

If $\operatorname{rank}(X)=s<r$, we have $\operatorname{rank}\left(X^{\prime}\right)<r$, which implies

$$
\left|\boldsymbol{\alpha}^{\prime}\right|=s, \quad \gamma^{\prime}=\emptyset, \quad \Lambda_{\boldsymbol{\beta}^{\prime}}\left(X^{\prime}\right)=0 .
$$

Thus, by denoting

$$
T:=\left\{\begin{array}{cc}
\boldsymbol{\alpha}^{\prime}, & \text { if } s<r \\
\boldsymbol{\alpha}^{\prime} \cup \boldsymbol{\beta}_{1}^{\prime}, & \text { if } s=r,
\end{array}\right.
$$


we can rewrite $X$ in (46) as

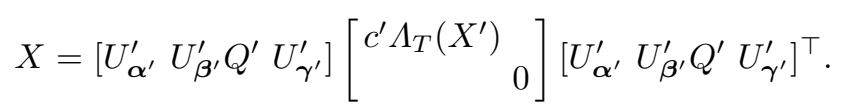

By the uniqueness theorem of eigenvalue decomposition in [48, Corollary 4.4.4], the entries in $\Lambda_{\Gamma}(X)$ and $c^{\prime} \Lambda_{T}\left(X^{\prime}\right)$ have the same values but different indices, and the columns of $U$ and $\left[U_{\boldsymbol{\alpha}^{\prime}}^{\prime} U_{\boldsymbol{\beta}^{\prime}}^{\prime} Q^{\prime} U_{\boldsymbol{\gamma}^{\prime}}^{\prime}\right]$ are the same but with different locations. Without loss of generality, we let

$$
\Gamma=T, \quad \Lambda_{\Gamma}(X)=c^{\prime} \Lambda_{T}\left(X^{\prime}\right), \quad U=\left[U_{\boldsymbol{\alpha}^{\prime}}^{\prime} U_{\boldsymbol{\beta}^{\prime}}^{\prime} Q^{\prime} U_{\boldsymbol{\gamma}^{\prime}}^{\prime}\right] .
$$

Then, we can rewrite $X^{\prime}$ as

$$
\begin{aligned}
& X^{\prime}=U^{\prime}\left[\begin{array}{lll}
\Lambda_{\boldsymbol{\alpha}^{\prime}}\left(X^{\prime}\right) & & \\
& \Lambda_{\boldsymbol{\beta}^{\prime}}\left(X^{\prime}\right) & \\
& & \Lambda_{\gamma^{\prime}}\left(X^{\prime}\right)
\end{array}\right]\left(U^{\prime}\right)^{\top} \\
& \stackrel{(11)}{=} U^{\prime}\left[\begin{array}{lll}
\Lambda_{\boldsymbol{\alpha}^{\prime}}\left(X^{\prime}\right) & & \\
& Q^{\prime} \Lambda_{\boldsymbol{\beta}^{\prime}}\left(X^{\prime}\right)\left(Q^{\prime}\right)^{\top} & \\
& & \Lambda_{\boldsymbol{\gamma}^{\prime}}\left(X^{\prime}\right)
\end{array}\right]\left(U^{\prime}\right)^{\top} \\
& \stackrel{(51)}{=} U\left[\begin{array}{lll}
\Lambda_{\boldsymbol{\alpha}^{\prime}}\left(X^{\prime}\right) & & \\
& \Lambda_{\boldsymbol{\beta}^{\prime}}\left(X^{\prime}\right) & \\
& & \Lambda_{\boldsymbol{\gamma}^{\prime}}\left(X^{\prime}\right)
\end{array}\right] U^{\top} \\
& \stackrel{(51)}{=} U\left[\begin{array}{ll}
\Lambda_{\Gamma}\left(X^{\prime}\right) & \\
& \Lambda_{\Gamma^{\perp}}\left(X^{\prime}\right)
\end{array}\right] U^{\top \stackrel{(51)}{=}} U\left[\begin{array}{ll}
\Lambda_{\Gamma}(X) / c^{\prime} & \\
& \Lambda_{\Gamma^{\perp}}\left(X^{\prime}\right)
\end{array}\right] U^{\top},
\end{aligned}
$$

which in combination with (50) yields

$$
\nabla f(X)=\frac{X-(X-\alpha \nabla f(X))}{\alpha}=\frac{X-X^{\prime}}{\alpha}=U\left[\begin{array}{r}
\rho \Lambda_{\Gamma}(X) \\
D
\end{array}\right] U^{\top},
$$

where

$$
\rho:=\frac{1-1 / c^{\prime}}{\alpha}, \quad D:=-\frac{\Lambda_{\Gamma^{\perp}}\left(X^{\prime}\right)}{\alpha} .
$$

This result proves $\nabla f(X)=\mathcal{M}_{X}\left(\rho \Lambda_{\Gamma}(X), D\right)$.

We now need to show that $\rho \leq 0$ and $\bar{\lambda}_{D} \leq(1 / \alpha-\rho) \lambda_{r}^{\text {abs }}(X)$, before which we require several facts. If $s<r$, then $\Lambda_{\boldsymbol{\beta}_{1}^{\prime}}\left(X^{\prime}\right)=0$ due to (48) and $\boldsymbol{\beta}_{1}^{\prime} \subseteq \boldsymbol{\beta}^{\prime}$, which results in

$$
\left\|\Lambda_{T}\left(X^{\prime}\right)\right\|_{F} \stackrel{(49)}{=}\left\|\Lambda_{\boldsymbol{\alpha}^{\prime}}\left(X^{\prime}\right)\right\|_{F}=\left\|\Lambda_{\boldsymbol{\alpha}^{\prime} \cup \boldsymbol{\beta}_{1}^{\prime}}\left(X^{\prime}\right)\right\|_{F} \stackrel{(47)}{=} \phi .
$$

When $s=r, T=\boldsymbol{\alpha}^{\prime} \cup \boldsymbol{\beta}_{1}^{\prime}$ from (49). Overall, we have

$$
\left\|\Lambda_{T}\left(X^{\prime}\right)\right\|_{F}=\phi
$$

which results in our first fact,

$$
\left\|\Lambda_{\Gamma}(X)\right\|_{F} \stackrel{(51)}{=} c^{\prime}\left\|\Lambda_{T}\left(X^{\prime}\right)\right\|_{F}=c^{\prime} \phi \stackrel{(47)}{=}\left\{\begin{array}{r}
\phi<1, \text { if } \phi<1 \\
1, \text { if } \phi \geq 1 .
\end{array}\right.
$$


The second fact is that

$$
\Gamma=\left\{\begin{array}{ll}
\boldsymbol{\alpha}^{\prime}, & r<s, \\
\boldsymbol{\alpha}^{\prime} \cup \boldsymbol{\beta}_{1}^{\prime}, & r=s,
\end{array}, \quad \Gamma^{\perp}= \begin{cases}\boldsymbol{\beta}^{\prime}, & r<s \\
\left(\boldsymbol{\beta}^{\prime} \backslash \boldsymbol{\beta}_{1}^{\prime}\right) \cup \gamma^{\prime}, & r=s\end{cases}\right.
$$

and thus, according to the definitions of $\boldsymbol{\alpha}^{\prime}, \boldsymbol{\beta}_{1}^{\prime}, \boldsymbol{\gamma}^{\prime}$, we obtain the third fact

$$
\min _{i \in \Gamma}\left|\lambda_{i}\left(X^{\prime}\right)\right| \geq \max _{i \in \Gamma^{\perp}}\left|\lambda_{i}\left(X^{\prime}\right)\right|
$$

Case 1) Suppose $X \in \operatorname{bd} \mathcal{C}_{1}$. Then, $\left\|\Lambda_{\Gamma}(X)\right\|_{F}=1$, which indicates $\phi \geq 1$ by (54) and hence $c^{\prime} \leq 1$ by (47). Therefore, $\rho \leq 0$ by (53). If $s=r$, the following chain of inequalities holds

$$
\begin{aligned}
(1 / \alpha-\rho) \lambda_{r}^{\mathrm{abs}}(X) & \stackrel{s=r}{=}(1 / \alpha-\rho) \min _{i \in \Gamma}\left|\lambda_{i}(X)\right| \\
& \stackrel{(51)}{=}(1 / \alpha-\rho) c^{\prime} \min _{i \in \Gamma}\left|\lambda_{i}\left(X^{\prime}\right)\right| \stackrel{(53)}{=} \min _{i \in \Gamma}\left|\lambda_{i}\left(X^{\prime}\right)\right| / \alpha \\
& \stackrel{(56)}{\geq} \max _{i \in \Gamma^{\perp}}\left|\lambda_{i}\left(X^{\prime}\right)\right| / \alpha \stackrel{(53)}{=} \bar{\lambda}_{D} .
\end{aligned}
$$

If $s<r$, then holds $\lambda_{r}^{\mathrm{abs}}(X)=0$ and

$$
D \stackrel{(53)}{=}-\Lambda_{\Gamma^{\perp}}\left(X^{\prime}\right) / \alpha \stackrel{(55)}{=} \Lambda_{\beta^{\prime}}\left(X^{\prime}\right) / \alpha \stackrel{(48)}{=} 0 .
$$

Thus, $\bar{\lambda}_{D}=0=(1 / \alpha-\rho) \lambda_{r}^{\text {abs }}(X)$. These results prove $(45)$.

Case 2) Suppose $X \in \operatorname{int} \mathcal{C}_{1}$. We must have $\left\|\Lambda_{\Gamma}(X)\right\|_{F}<1$, which implies $\phi<1$ by (54) and hence $c^{\prime}=1$ by (47). This result yields $\rho=0$. The rest of the proof is the same as that of Case 2).

Theorem 5.2 For any $X \in \mathbb{S}^{n}(r) \cap \mathcal{C}_{2}$ with the same EVD as in (6), denote $s:=\operatorname{rank}(X)=|\Gamma|$ and let $\boldsymbol{\eta}_{+}, \boldsymbol{\eta}_{ \pm}, \boldsymbol{\eta}_{-}, \boldsymbol{\eta}_{=}$be defined as in (37). We have the following results.

(i) $X$ is an F-stationary point of (1) if and only if

$$
\nabla f(X) \in\left\{\begin{array}{l}
\left\{\mathcal{N}_{X}(A, 0, C, 0): A \in \mathbb{S}_{-}^{\left|\boldsymbol{\eta}_{+}\right|}, C \in \mathbb{S}_{+}^{\left|\boldsymbol{\eta}_{-}\right|}\right\}, \quad s<r, \\
\left\{\mathcal{N}_{X}(A, 0, C, D): \begin{array}{l}
A \in \mathbb{S}_{-}^{\left|\boldsymbol{\eta}_{+}\right|}, C \in \mathbb{S}_{+}^{\left|\boldsymbol{\eta}_{-}\right|}, \\
D \in \mathbb{S}^{\left|\boldsymbol{\eta}_{-}\right|}
\end{array}\right\}, s=r .
\end{array}\right.
$$

(ii) $X$ is an $\alpha$-stationary point of (1) for a given $\alpha>0$ if and only if

$$
\nabla f(X) \in\left\{\mathcal{N}_{X}(A, 0, C, D): \begin{array}{l}
A \in \mathbb{S}_{-}^{\left|\boldsymbol{\eta}_{+}\right|}, C \in \mathbb{S}_{+}^{\left|\boldsymbol{\eta}_{-}\right|} \\
D \in \mathbb{S}^{\left|\boldsymbol{\eta}_{-1}\right|} \text { with } \bar{\lambda}_{D} \leq \frac{1}{\alpha} \lambda_{r}^{\text {abs }}(X)
\end{array}\right\}
$$

where $\bar{\lambda}_{D}:=\max _{i \in\{1, \cdots, n-s\}}\left|\lambda_{i}(D)\right|$. 
Proof. (i) By Lemma 4.1, Proposition 4.1 (ii) and Theorem 4.1, we can obtain the formula of $N_{\mathbb{S}^{n}(r) \cap \mathcal{C}_{2}}^{F}(X)$, which enables us to derive the conclusion.

(ii) Sketch of the proof: For the 'If' part, we need to show that

$$
\nabla f(X) \in \Theta_{2} \Longrightarrow X \in \Pi_{\mathbb{S}^{n}(r) \cap \mathcal{C}_{2}}(X-\alpha \nabla f(X)), \forall X \in \mathbb{S}^{n}(r) \cap \mathcal{C}_{2},
$$

where $\Theta_{2}$ is the set of the right-hand side of (58). For the 'Only if' part, we need to show that

$$
X \in \Pi_{\mathbb{S}^{n}(r) \cap \mathcal{C}_{2}}(X-\alpha \nabla f(X)) \quad \Longrightarrow \quad \nabla f(X) \in \Theta_{2} .
$$

To do this, we first prove that $X$ and $X^{\prime}:=X-\alpha \nabla f(X)$ has a similar decomposition to

$$
X=U\left[\begin{array}{cc}
\Lambda_{\Gamma}(X) & \\
& 0
\end{array}\right] U^{\top}, \quad X^{\prime}=U\left[\begin{array}{ll}
\Lambda_{1} & \\
& \Lambda_{2}
\end{array}\right] U^{\top},
$$

where $\Lambda_{1}, \Lambda_{2}$ will be specified later, which results in $\nabla f(X)=\mathcal{M}_{X}(H, D)$. We then prove that $H$ takes the form

$$
H=\left[\begin{array}{lll}
A & & \\
& 0 & \\
& & C
\end{array}\right],
$$

which yields $\nabla f(X)=\mathcal{N}_{X}(A, 0, C, D)$. We complete the proof by verifying $\bar{\lambda}_{D} \leq \frac{1}{\alpha} \lambda_{r}^{\mathrm{abs}}(X)$.

'If' part: For any $X \in \mathbb{S}^{n}(r) \cap \mathcal{C}_{2}$, it follows from $X \in \mathcal{C}_{2}$ and (39) that

$$
\Gamma=\boldsymbol{\eta}_{+} \cup \boldsymbol{\eta}_{ \pm} \cup \boldsymbol{\eta}_{-} .
$$

Since $\nabla f(X) \in \Theta_{2}$, there is an $A \in \mathbb{S}_{-}^{\left|\boldsymbol{\eta}_{+}\right|}, C \in \mathbb{S}_{+}^{\left|\boldsymbol{\eta}_{-}\right|}$and $D \in \mathbb{S}_{+}^{\left|\boldsymbol{\eta}_{=}\right|}$with $\bar{\lambda}_{D} \leq \frac{1}{\alpha} \lambda_{r}^{\mathrm{abs}}(X)$ such that

$$
\nabla f(X)=\mathcal{N}_{X}(A, 0, C, D) \stackrel{(36,38,59)}{=} \mathcal{M}_{X}(E, 0), \text { where } E=:\left[\begin{array}{lll}
A & & \\
& 0 & \\
& & C
\end{array}\right],
$$

which contributes to

$$
\begin{aligned}
X-\alpha \nabla f(X) & =\mathcal{M}_{X}\left(\Lambda_{\Gamma}(X), 0\right)-\alpha \mathcal{M}_{X}(E, D) \\
& =\mathcal{M}_{X}\left(\Lambda_{\Gamma}(X)-\alpha E,-\alpha D\right)
\end{aligned}
$$

and

$$
\Lambda_{\Gamma}(X)-\alpha E=\left[\begin{array}{lll}
\Lambda_{\boldsymbol{\eta}_{+}}(X)-\alpha A & & \\
& \Lambda_{\boldsymbol{\eta}_{ \pm}}(X) & \\
& & \Lambda_{\boldsymbol{\eta}_{-}}(X)-\alpha C
\end{array}\right] .
$$

As $A \in \mathbb{S}_{-}^{\left|\boldsymbol{\eta}_{+}\right|}, C \in \mathbb{S}_{+}^{\left|\boldsymbol{\eta}_{-}\right|}$and $\alpha>0$, it follows that

$$
\begin{aligned}
& \lambda_{i}(X-\alpha \nabla f(X))=\lambda_{i}\left(\Lambda_{\Gamma}(X)-\alpha E\right) \\
& =\left\{\begin{array}{rlrl}
t-\alpha \lambda_{i}(A) & \geq t, & & i \in \boldsymbol{\eta}_{+}, \\
\lambda_{i}(X) & \in(-t, 0) \cup(0, t), & i \in \boldsymbol{\eta}_{ \pm}, \\
-t-\alpha \lambda_{i}(C) & \leq-t, & & i \in \boldsymbol{\eta}_{-} .
\end{array}\right.
\end{aligned}
$$


If $s<r$, then $\lambda_{r}^{\text {abs }}(X)=0$, making $D=0$. If $s=r$, by the definitions (37) of $\boldsymbol{\eta}_{+}, \boldsymbol{\eta}_{ \pm}, \boldsymbol{\eta}_{-}$, it is easy to see that $\lambda_{r}^{\text {abs }}(X)=\min _{i \in \boldsymbol{\eta}_{ \pm}}\left|\lambda_{i}(X)\right|$, which together with $\bar{\lambda}_{D} \leq \frac{1}{\alpha} \lambda_{r}^{\text {abs }}(X)$ suffices to show that

$$
\begin{aligned}
\max _{i \in\{1, \cdots, n-s\}}\left|\lambda_{i}(-\alpha D)\right| & =\alpha \max _{i \in\{1, \cdots, n-s\}}\left|\lambda_{i}(D)\right|=\alpha \bar{\lambda}_{D} \\
& \leq \lambda_{r}^{\text {abs }}(X)=\min _{i \in \boldsymbol{\eta}_{ \pm}}\left|\lambda_{i}(X)\right| \\
& \stackrel{(61)}{=} \min _{i \in \boldsymbol{\eta}_{ \pm}}\left|\lambda_{i}(X-\alpha \nabla f(X))\right| \\
& =\min _{i \in \Gamma}\left|\lambda_{i}(X-\alpha \nabla f(X))\right| .
\end{aligned}
$$

Both cases allow us to derive

$$
\Pi_{\mathbb{S}^{n}(r)}\left(\mathcal{M}_{X}\left(\Lambda_{\Gamma}(X)-\alpha E,-\alpha D\right)\right)=\mathcal{M}_{X}\left(\Lambda_{\Gamma}(X)-\alpha E, 0\right)
$$

and hence to obtain

$$
\begin{aligned}
\Pi_{\mathbb{S}^{n}(r) \cap \mathcal{C}_{2}}(X-\alpha \nabla f(X)) & \stackrel{(22)}{=} \Pi_{\mathcal{C}_{2}} \Pi_{\mathbb{S}^{n}(r)}(X-\alpha \nabla f(X)) \\
& \stackrel{(60)}{=} \Pi_{\mathcal{C}_{2}} \Pi_{\mathbb{S}^{n}(r)}\left(\mathcal{M}_{X}\left(\Lambda_{\Gamma}(X)-\alpha E,-\alpha D\right)\right) \\
& =\Pi_{\mathcal{C}_{2}}\left(\mathcal{M}_{X}\left(\Lambda_{\Gamma}(X)-\alpha E, 0\right)\right) \\
& \stackrel{(61)}{=} X .
\end{aligned}
$$

Therefore, $X$ is an $\alpha$-stationary point, which proves the 'If' part.

'Only if' part: For any $X \in \boldsymbol{\Pi}_{\mathbb{S}^{n}(r) \cap \mathcal{C}_{2}}(X-\alpha \nabla f(X))$, let

$$
X^{\prime}:=X-\alpha \nabla f(X)=U^{\prime} \operatorname{Diag}\left(\lambda\left(X^{\prime}\right)\right)\left(U^{\prime}\right)^{\top}
$$

and $\boldsymbol{\alpha}^{\prime}, \boldsymbol{\beta}^{\prime}, \boldsymbol{\gamma}^{\prime}$ be defined as in (8) and $Q^{\prime}$ be defined as in (10), in which $X$ is replaced by $X^{\prime}$. It follows from (23) that for any $X \in \Pi_{\mathbb{S}^{n}(r) \cap \mathcal{C}_{2}}\left(X^{\prime}\right)$, there is a $Q^{\prime}$ satisfying (10) and a $\boldsymbol{\beta}_{2}^{\prime} \subseteq \boldsymbol{\beta}^{\prime},\left|\boldsymbol{\beta}_{2}^{\prime}\right|=r-\left|\boldsymbol{\alpha}^{\prime}\right|$ such that

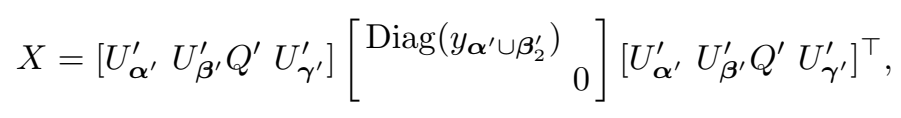

where

$$
y \in \mathbb{R}^{n} \text { with } \quad y_{i}=\operatorname{sign}\left(\lambda_{i}\left(X^{\prime}\right)\right) \min \left\{\left|\lambda_{i}\left(X^{\prime}\right)\right|, t\right\}, i=1, \ldots, n .
$$

Recall that $X$ has the same EVD as in (6), i.e.,

$$
X=U\left[\begin{array}{cc}
\Lambda_{\Gamma}(X) & \\
& 0
\end{array}\right] U^{\top} .
$$

If $\operatorname{rank}(X)=s<r$, we have $\operatorname{rank}\left(X^{\prime}\right)<r$, which implies

$$
\left|\boldsymbol{\alpha}^{\prime}\right|=s, \quad \gamma^{\prime}=\emptyset, \quad \Lambda_{\boldsymbol{\beta}^{\prime}}\left(X^{\prime}\right)=0 .
$$


Similarly as the proof of (51), by the uniqueness theorem of eigenvalue decomposition in [48, Corollary 4.4.4], we can let

$$
\Gamma=\left\{\begin{array}{c}
\boldsymbol{\alpha}^{\prime}, \quad \text { if } s<r, \\
\boldsymbol{\alpha}^{\prime} \cup \boldsymbol{\beta}_{2}^{\prime}, \text { if } s=r,
\end{array} \quad \Lambda_{\Gamma}(X)=\operatorname{Diag}\left(y_{\Gamma}\right), \quad U=\left[\begin{array}{ll}
U_{\boldsymbol{\alpha}^{\prime}}^{\prime} & U_{\boldsymbol{\beta}^{\prime}}^{\prime}
\end{array} Q^{\prime} U_{\boldsymbol{\gamma}^{\prime}}^{\prime}\right]\right.
$$

and rewrite (62) as

$$
X=\left[\begin{array}{llll}
U_{\boldsymbol{\alpha}^{\prime}}^{\prime} & U_{\boldsymbol{\beta}^{\prime}}^{\prime} & Q^{\prime} & U_{\boldsymbol{\gamma}^{\prime}}^{\prime}
\end{array}\right]\left[\begin{array}{rr}
\operatorname{Diag}\left(y_{\Gamma}\right) & \\
& 0
\end{array}\right]\left[\begin{array}{lll}
U_{\boldsymbol{\alpha}^{\prime}}^{\prime} & U_{\boldsymbol{\beta}^{\prime}}^{\prime} Q^{\prime} & U_{\boldsymbol{\gamma}^{\prime}}^{\prime}
\end{array}\right]^{\top} .
$$

Then, we can rewrite $X^{\prime}$ as

$$
\begin{aligned}
& X^{\prime}=U^{\prime}\left[\begin{array}{lll}
\Lambda_{\alpha^{\prime}}\left(X^{\prime}\right) & & \\
& \Lambda_{\boldsymbol{\beta}^{\prime}}\left(X^{\prime}\right) & \\
& & \Lambda_{\gamma^{\prime}}\left(X^{\prime}\right)
\end{array}\right]\left(U^{\prime}\right)^{\top} \\
& \stackrel{(11)}{=} U^{\prime}\left[\begin{array}{lll}
\Lambda_{\boldsymbol{\alpha}^{\prime}}\left(X^{\prime}\right) & & \\
& Q^{\prime} \Lambda_{\boldsymbol{\beta}^{\prime}}\left(X^{\prime}\right)\left(Q^{\prime}\right)^{\top} & \\
& & \Lambda_{\boldsymbol{\gamma}^{\prime}}\left(X^{\prime}\right)
\end{array}\right]\left(U^{\prime}\right)^{\top} \\
& \stackrel{(65)}{=} U\left[\begin{array}{lll}
\Lambda_{\boldsymbol{\alpha}^{\prime}}\left(X^{\prime}\right) & & \\
& \Lambda_{\boldsymbol{\beta}^{\prime}}\left(X^{\prime}\right) & \\
& & \Lambda_{\boldsymbol{\gamma}^{\prime}}\left(X^{\prime}\right)
\end{array}\right] U^{\top} \\
& \stackrel{(65)}{=} U\left[\begin{array}{ll}
\Lambda_{\Gamma}\left(X^{\prime}\right) & \\
& \Lambda_{\Gamma^{\perp}}\left(X^{\prime}\right)
\end{array}\right] U^{\top} \text {. }
\end{aligned}
$$

The above two equations contribute to

$$
\nabla f(X)=\frac{X-(X-\alpha \nabla f(X))}{\alpha}=\frac{X-X^{\prime}}{\alpha}=U\left[\begin{array}{ll}
H & \\
& D
\end{array}\right] U^{\top},
$$

where

$$
H:=\frac{\operatorname{Diag}\left(y_{\Gamma}\right)-\Lambda_{\Gamma}\left(X^{\prime}\right)}{\alpha}, \quad D:=-\frac{\Lambda_{\Gamma^{\perp}}\left(X^{\prime}\right)}{\alpha} .
$$

Now, let us consider $H$. For any $i \in \Gamma$, we have

$$
\begin{aligned}
\alpha H_{i i} & =\lambda_{i}(X)-\lambda_{i}\left(X^{\prime}\right) \\
& \stackrel{(63)}{=} \operatorname{sign}\left(\lambda_{i}\left(X^{\prime}\right)\right) \min \left\{\left|\lambda_{i}\left(X^{\prime}\right)\right|, t\right\}-\lambda_{i}\left(X^{\prime}\right) \\
& =\left\{\begin{array}{rr}
-t-\lambda_{i}\left(X^{\prime}\right) & \geq 0, \\
t-\lambda_{i}\left(X^{\prime}\right) & \leq 0, \quad \lambda_{i}\left(X^{\prime}\right) \leq-t, \\
& =0,0<\left|\lambda_{i}\left(X^{\prime}\right)\right|<t .
\end{array}\right.
\end{aligned}
$$

This result implies that

$$
\begin{array}{lll}
\left\{i \in \Gamma: \lambda_{i}\left(X^{\prime}\right) \geq t\right\} & =\left\{i \in \Gamma: \lambda_{i}(X)=t\right\} & =\boldsymbol{\eta}_{+}, \\
\left\{i \in \Gamma: \lambda_{i}\left(X^{\prime}\right) \leq-t\right\} & =\left\{i \in \Gamma: \lambda_{i}(X)=-t\right\} & =\boldsymbol{\eta}_{-} \\
\left\{i \in \Gamma: 0<\left|\lambda_{i}\left(X^{\prime}\right)\right|<t\right\} & =\left\{i \in \Gamma: \lambda_{i}(X)=\lambda_{i}\left(X^{\prime}\right) \neq 0\right\}=\boldsymbol{\eta}_{ \pm}
\end{array}
$$


Based on these equations, define

$$
\begin{aligned}
A & :=\left[\operatorname{Diag}\left(y_{\boldsymbol{\eta}_{+}}\right)-\Lambda_{\boldsymbol{\eta}_{+}}\left(X^{\prime}\right)\right] / \alpha=\left[\Lambda_{\boldsymbol{\eta}_{+}}(X)-\Lambda_{\boldsymbol{\eta}_{+}}\left(X^{\prime}\right)\right] / \alpha \stackrel{(69)}{\in} \mathbb{S}_{-}^{\left|\boldsymbol{\eta}_{+}\right|}, \\
C & :=\left[\operatorname{Diag}\left(y_{\boldsymbol{\eta}_{-}}\right)-\Lambda_{\boldsymbol{\eta}_{-}}\left(X^{\prime}\right)\right] / \alpha=\left[\Lambda_{\boldsymbol{\eta}_{-}}(X)-\Lambda_{\boldsymbol{\eta}_{-}}\left(X^{\prime}\right)\right] / \alpha \stackrel{(69)}{\in} \mathbb{S}_{+}^{\left|\boldsymbol{\eta}_{-}\right|} .
\end{aligned}
$$

Therefore, $\nabla f(X)$ can be rewritten as

$$
\begin{aligned}
& \nabla f(X)=\left[U_{\boldsymbol{\eta}_{+}} U_{\boldsymbol{\eta}_{ \pm}} U_{\boldsymbol{\eta}_{-}} U_{\boldsymbol{\eta}_{=}}\right]\left[\begin{array}{llll}
A & & & \\
& 0 & & \\
& & C & \\
& & D
\end{array}\right]\left[U_{\boldsymbol{\eta}_{+}} U_{\boldsymbol{\eta}_{ \pm}} U_{\boldsymbol{\eta}_{-}} U_{\boldsymbol{\eta}_{=}}\right]^{\top} \\
& =\mathcal{N}_{X}(A, 0, C, D) \text { with } A \in \mathbb{S}_{-}^{\left|\boldsymbol{\eta}_{+}\right|}, \quad C \in \mathbb{S}_{+}^{\left|\boldsymbol{\eta}_{-}\right|} \text {. }
\end{aligned}
$$

Similarly, we also have (55), where $\boldsymbol{\beta}_{1}^{\prime}$ is replaced by $\boldsymbol{\beta}_{2}^{\prime}$, and (56). If $s=r$, then

$$
\begin{aligned}
\bar{\lambda}_{D} & \stackrel{(68)}{=} \max _{i \in \Gamma^{\perp}}\left|\lambda_{i}\left(X^{\prime}\right)\right| / \alpha \\
& \stackrel{(56)}{\leq} \min _{i \in \Gamma}\left|\lambda_{i}\left(X^{\prime}\right)\right| / \alpha \stackrel{(70)}{=} \min _{i \in \boldsymbol{\eta}_{+} \cup \boldsymbol{\eta}_{ \pm} \cup \boldsymbol{\eta}_{-}}\left|\lambda_{i}\left(X^{\prime}\right)\right| / \alpha \\
& =\min _{i \in \boldsymbol{\eta}_{ \pm}}\left|\lambda_{i}\left(X^{\prime}\right)\right| / \alpha \stackrel{(70)}{=} \min _{i \in \boldsymbol{\eta}_{ \pm}}\left|\lambda_{i}(X)\right| / \alpha=\lambda_{r}^{\mathrm{abs}}(X) / \alpha .
\end{aligned}
$$

If $s<r$, it holds that $\lambda_{r}^{\mathrm{abs}}(X)=0$ and

$$
D \stackrel{(68)}{=}-\Lambda_{\Gamma^{\perp}}\left(X^{\prime}\right) / \alpha \stackrel{(55)}{=} \Lambda_{\beta^{\prime}}\left(X^{\prime}\right) / \alpha \stackrel{(64)}{=} 0 .
$$

Thus, $\bar{\lambda}_{D}=0=\lambda_{r}^{\mathrm{abs}}(X) / \alpha$. These results prove (58).

Theorem 5.3 For any $X \in \mathbb{S}^{n}(r) \cap \mathcal{C}_{3}$ with the same EVD as in (6) and denoting $s:=\operatorname{rank}(X)=|\Gamma|$, we have the following results.

(i) $X$ is an F-stationary point of (1) if and only if

$$
\nabla f(X) \in \begin{cases}\left\{\mathcal{M}_{X}\left(\rho I_{s}, \rho I_{n-s}+D\right): \rho \in \mathbb{R}, D \in \mathbb{S}_{+}^{n-s}\right\}, s<r, \\ \left\{\mathcal{M}_{X}\left(\rho I_{s}, D\right): \rho \in \mathbb{R}, D \in \mathbb{S}^{n-s}\right\}, & s=r .\end{cases}
$$

(ii) $X$ is an $\alpha$-stationary point of (1) for a given $\alpha>0$ if and only if

$$
\nabla f(X) \in\left\{\mathcal{M}_{X}\left(\rho I_{s}, \rho I_{n-s}+D\right): \begin{array}{l}
\rho \in \mathbb{R}, D \in \mathbb{S}^{n-s} \\
\\
\text { with } \underline{\lambda}_{D} \geq-\lambda_{r}(X) / \alpha
\end{array}\right\}
$$

where $\underline{\lambda}_{D}:=\min _{i \in\{1, \cdots, n-s\}} \lambda_{i}(D)$. 
Proof. (i) By Lemma 4.1, Proposition 4.1 (iii) and the sum rule of Theorem 4.1, we can obtain the formula of $N_{\mathbb{S}^{n}(r) \cap \mathcal{C}_{3}}^{F}(X)$, which can be used to derive the conclusion.

(ii) Sketch of the proof: For the 'If' part, we need to show that

$$
\nabla f(X) \in \Theta_{3} \quad \Longrightarrow \quad X \in \Pi_{\mathbb{S}^{n}(r) \cap \mathcal{C}_{3}}(X-\alpha \nabla f(X)), \forall X \in \mathbb{S}^{n}(r) \cap \mathcal{C}_{3},
$$

where $\Theta_{3}$ is the set of the right-hand side of (72). To do so, we prove two equations

$$
\Pi_{\mathbb{S}^{n}(r) \cap \mathcal{C}_{3}}(X-\alpha \nabla f(X))=\Pi_{\mathcal{C}_{3}}\left(\mathcal{M}_{X}\left(\Lambda_{0}, 0\right)\right)=X
$$

where $\Lambda_{0}$ will be specified later. For the 'Only if' part, we need to show that

$$
X \in \Pi_{\mathbb{S}^{n}(r) \cap \mathcal{C}_{3}}(X-\alpha \nabla f(X)) \Longrightarrow \nabla f(X) \in \Theta_{3} .
$$

To do this, we first prove that $X$ and $X^{\prime}:=X-\alpha \nabla f(X)$ has a similar decomposition to

$$
X=U\left[\begin{array}{cc}
\Lambda_{\Gamma}(X) & \\
& 0
\end{array}\right] U^{\top}, \quad X^{\prime}=U\left[\begin{array}{ll}
\Lambda_{1} & \\
& \Lambda_{2}
\end{array}\right] U^{\top},
$$

where $\Lambda_{1}, \Lambda_{2}$ will be specified later, which yields $\nabla f(X)=\mathcal{M}_{X}\left(H, \rho I_{n-s}+D\right)$. We then prove that $H=\rho I_{s}$ and $\underline{\lambda}_{D} \geq-\lambda_{r}(X) / \alpha$.

'If' part: For any $X \in \mathbb{S}^{n}(r) \cap \mathcal{C}_{3}$, it follows from $\nabla f(X) \in \Theta_{3}$ that there is a $\rho \in \mathbb{R}, D \in \mathbb{S}^{n-s}$ with $\underline{\lambda}_{D} \geq-\lambda_{r}(X) / \alpha$ satisfying

$$
\nabla f(X)=\mathcal{M}_{X}\left(\rho I_{s}, \rho I_{n-s}+D\right),
$$

which suffices to show that

$$
\begin{aligned}
& \Pi_{\mathbb{S}^{n}(r) \cap \mathcal{C}_{3}}(X-\alpha \nabla f(X)) \\
= & \Pi_{\mathbb{S}^{n}}(r) \cap \mathcal{C}_{3}\left(\mathcal{M}_{X}\left(\Lambda_{\Gamma}(X), 0\right)-\alpha \mathcal{M}_{X}\left(\rho I_{s}, \rho I_{n-s}+D\right)\right) \\
= & \Pi_{\mathbb{S}^{n}(r) \cap \mathcal{C}_{3}}\left(\mathcal{M}_{X}\left(\Lambda_{\Gamma}(X)-\alpha \rho I_{s},-\alpha D-\alpha \rho I_{n-s}\right)\right) \\
\stackrel{(32)}{=} & \Pi_{\mathcal{C}_{3}} \Pi_{\mathcal{L}_{r}}\left(\mathcal{M}_{X}\left(\Lambda_{\Gamma}(X)-\alpha \rho I_{s},-\alpha D-\alpha \rho I_{n-s}\right)\right) \\
= & \Pi_{\mathcal{C}_{3}}\left(\mathcal{M}_{X}\left(\Lambda_{\Gamma}(X)-\alpha \rho I_{s}, 0\right)\right),
\end{aligned}
$$

where the last equality holds because

$$
\begin{aligned}
\max _{i \in\{1, \cdots, n-s\}} \lambda_{i}\left(-\alpha D-\alpha \rho I_{n-s}\right) & =-\alpha \min _{i \in\{1, \cdots, n-s\}} \lambda_{i}(D)-\alpha \rho \\
& =-\alpha \underline{\lambda}_{D}-\alpha \rho \leq \lambda_{r}(X)-\alpha \rho \\
& \leq \min _{i \in \Gamma} \lambda_{i}(X)-\alpha \rho \\
& =\min _{i \in \Gamma} \lambda_{i}\left(X-\alpha \rho I_{s}\right) .
\end{aligned}
$$

Now, we need to prove $\Pi_{\mathcal{C}_{3}}\left(\mathcal{M}_{X}\left(\Lambda_{\Gamma}(X)-\alpha \rho I_{s}, 0\right)\right)=X$. From Lemma 3.1, this fact can be verified by proving the following result

$$
\lambda(X)=\left[\begin{array}{c}
\lambda_{\Gamma}(X) \\
0
\end{array}\right]=\operatorname{argmin}_{z \in \mathcal{K}_{3}}\left\|z-\left[\begin{array}{c}
\lambda_{\Gamma}(X)-\alpha \rho e \\
0
\end{array}\right]\right\|_{2}^{2},
$$


where $e$ is a vector with all elements being one. In fact, $\mathcal{K}_{3}$ is convex; thus, the right-hand side has a unique optimal solution. Since $X \in \mathcal{C}_{3}$, it follows that $\lambda(X) \in \mathcal{K}_{3}$. One can prove that $\lambda(X)$ is the unique solution by verifying the Karush-Kuhn-Tucker(KKT)-system of the above problem, where the Lagrange multipliers for $z \geq 0$ and $e^{\top} z=0$ are $\left[0 \alpha \rho e^{\top}\right]^{\top}$ and $-\alpha \rho$, respectively. Overall,

$$
\Pi_{\mathbb{S}^{n}(r) \cap \mathcal{C}_{3}}(X-\alpha \nabla f(X)) \stackrel{(73)}{=} \Pi_{\mathcal{C}_{3}}\left(\mathcal{M}_{X}\left(\Lambda_{\Gamma}(X)-\alpha \rho I_{s}, 0\right)\right)=X,
$$

which means $X$ is an $\alpha$-stationary point.

'Only if' part: For any $X \in \boldsymbol{\Pi}_{\mathbb{S}^{n}}(r) \cap \mathcal{C}_{3}(X-\alpha \nabla f(X))$, let

$$
X^{\prime}:=X-\alpha \nabla f(X)=U^{\prime} \operatorname{Diag}\left(\lambda\left(X^{\prime}\right)\right)\left(U^{\prime}\right)^{\top} .
$$

Let $\boldsymbol{a}^{\prime}, \boldsymbol{b}^{\prime}, \boldsymbol{c}^{\prime}$ be defined as in (24-26), in which $X$ is replaced by $X^{\prime}$. It follows from (33) that for any $X \in \boldsymbol{\Pi}_{\mathbb{S}^{n}(r) \cap \mathcal{C}_{3}}\left(X^{\prime}\right)$, there exists a $P^{\prime} \in \mathcal{O}^{\left|\boldsymbol{b}^{\prime}\right|}$ and a $\boldsymbol{b}_{3}^{\prime} \subseteq \boldsymbol{b}^{\prime},\left|\boldsymbol{b}_{2}^{\prime}\right|=r-\left|\boldsymbol{a}^{\prime}\right|$ such that

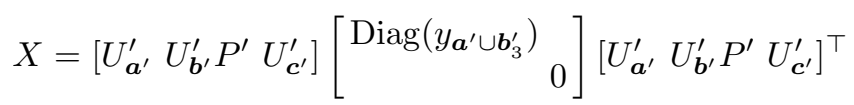

where

$$
y \in \mathbb{R}^{n} \text { with } \quad y_{i}=\max \left\{\lambda_{i}\left(X^{\prime}\right)-\tau, 0\right\}, i=1, \ldots, n
$$

and $\tau:=\frac{1}{\varrho}\left(\sum_{j=1}^{\varrho} \lambda_{j}\left(X^{\prime}\right)-1\right), \varrho:=\max \left\{j: \lambda_{j}\left(X^{\prime}\right)>\frac{1}{j}\left(\sum_{i=1}^{j} \lambda_{i}\left(X^{\prime}\right)-1\right)\right\}$. Recall that $X$ has the same EVD as in (6), i.e.,

$$
X=U\left[\begin{array}{cc}
\Lambda_{\Gamma}(X) & \\
& 0
\end{array}\right] U^{\top} .
$$

If $s<r$, we have $y_{i}=0, \forall i \in \boldsymbol{b}_{3}^{\prime}$, which together with (75) indicates

$$
\lambda_{r}\left(X^{\prime}\right)=\lambda_{i}\left(X^{\prime}\right) \leq \tau, \forall i \in \boldsymbol{b}^{\prime} .
$$

If $s=r$, we have $y_{i}>0, \forall i \in \boldsymbol{b}_{3}^{\prime}$, which together with (75) indicates

$$
\lambda_{r}\left(X^{\prime}\right)=\lambda_{i}\left(X^{\prime}\right)>\tau, \forall i \in \boldsymbol{b}^{\prime} .
$$

Similarly as the proof of (51), by the uniqueness theorem of eigenvalue decomposition in [48, Corollary 4.4.4], we can let

$$
\Gamma=\left\{\begin{array}{c}
\boldsymbol{a}^{\prime}, \quad \text { if } s<r, \\
\boldsymbol{a}^{\prime} \cup \boldsymbol{b}_{3}^{\prime}, \text { if } s=r,
\end{array} \quad \Lambda_{\Gamma}(X)=\operatorname{Diag}\left(y_{\Gamma}\right), \quad U=\left[U_{\boldsymbol{a}^{\prime}}^{\prime} U_{\boldsymbol{b}^{\prime}}^{\prime} P^{\prime} U_{\boldsymbol{c}^{\prime}}^{\prime}\right]\right.
$$

and can rewrite $X$ in (74) as

$$
X=\left[\begin{array}{llll}
U_{\boldsymbol{a}^{\prime}}^{\prime} & U_{\boldsymbol{b}^{\prime}}^{\prime} & P^{\prime} & U_{\boldsymbol{c}^{\prime}}^{\prime}
\end{array}\right]\left[\begin{array}{rr}
\operatorname{Diag}\left(y_{T}\right) & \\
& 0
\end{array}\right]\left[\begin{array}{lll}
U_{\boldsymbol{a}^{\prime}}^{\prime} & U_{\boldsymbol{b}^{\prime}}^{\prime} P^{\prime} & U_{\boldsymbol{c}^{\prime}}^{\prime}
\end{array}\right]^{\top} .
$$


Then, $X^{\prime}$ can be written as

$$
\begin{aligned}
& X^{\prime}=U^{\prime}\left[\begin{array}{lll}
\Lambda_{a^{\prime}}\left(X^{\prime}\right) & & \\
& \Lambda_{b^{\prime}}\left(X^{\prime}\right) & \\
& & \Lambda_{c^{\prime}}\left(X^{\prime}\right)
\end{array}\right]\left(U^{\prime}\right)^{\top} \\
& \stackrel{(28)}{=} U^{\prime}\left[\begin{array}{lll}
\Lambda_{\boldsymbol{a}^{\prime}}\left(X^{\prime}\right) & & \\
& P^{\prime} \Lambda_{\boldsymbol{b}^{\prime}}\left(X^{\prime}\right)\left(P^{\prime}\right)^{\top} & \\
& & \Lambda_{\boldsymbol{c}^{\prime}}\left(X^{\prime}\right)
\end{array}\right]\left(U^{\prime}\right)^{\top} \\
& \stackrel{(78)}{=} U\left[\begin{array}{lll}
\Lambda_{\boldsymbol{a}^{\prime}}\left(X^{\prime}\right) & & \\
& \Lambda_{\boldsymbol{b}^{\prime}}\left(X^{\prime}\right) & \\
& & \Lambda_{\boldsymbol{c}^{\prime}}\left(X^{\prime}\right)
\end{array}\right] U^{\top} \\
& =U\left[\begin{array}{ll}
\Lambda_{\Gamma}\left(X^{\prime}\right) & \\
& \Lambda_{\Gamma^{\perp}}\left(X^{\prime}\right)
\end{array}\right] U^{\top} .
\end{aligned}
$$

These two equations yield

$$
\nabla f(X)=\frac{X-X^{\prime}}{\alpha}=U\left[\begin{array}{ll}
H & \\
& \rho I_{n-s}+D
\end{array}\right] U^{\top}
$$

with

$$
\rho:=-\tau / \alpha, \quad H:=\frac{\operatorname{Diag}\left(y_{\Gamma}\right)-\Lambda_{\Gamma}\left(X^{\prime}\right)}{\alpha}, \quad D:=-\frac{\Lambda_{\Gamma^{\perp}}\left(X^{\prime}\right)}{\alpha}-\rho I .
$$

For any $i \in \Gamma$, we have $\lambda_{i}(X) \neq 0$, which implies

$$
0 \neq \lambda_{i}(X)=y_{i}=\max \left\{\lambda_{i}\left(X^{\prime}\right)-\tau, 0\right\}=\lambda_{i}\left(X^{\prime}\right)-\tau \stackrel{(75)}{>} 0, \quad i \in \Gamma .
$$

Therefore, we have

$$
H \stackrel{(82)}{=} \frac{\Lambda_{\Gamma}\left(X^{\prime}\right)-\tau I_{s}-\Lambda_{\Gamma}\left(X^{\prime}\right)}{\alpha}=\rho I_{s} .
$$

This result proves $\nabla f(X)=\mathcal{M}_{X}\left(\rho I_{s}, \rho I_{n-s}+D\right)$. The final step is to estimate $\underline{\lambda}_{D}$. If $s=r$, the definitions (24-26) of $\boldsymbol{a}^{\prime}, \boldsymbol{b}^{\prime}, \boldsymbol{c}^{\prime}$ yield

$$
\max _{i \in \Gamma^{\perp}} \lambda_{i}\left(X^{\prime}\right) \leq \min _{i \in \Gamma} \lambda_{i}\left(X^{\prime}\right),
$$

which is beneficial for the following chain of inequalities

$$
\begin{array}{r}
\underline{\lambda}_{D}=\min _{i \in\{1, \cdots, n-s\}} \lambda_{i}(D) \stackrel{(81)}{=}-\max _{i \in \Gamma^{\perp}} \lambda_{i}\left(X^{\prime}\right) / \alpha-\rho \\
\stackrel{\stackrel{(82)}{=}}{\stackrel{(81)}{=}}-\min _{i \in \Gamma} \lambda_{i}\left(X^{\prime}\right) / \alpha-\rho \stackrel{(82)}{=}-\min _{i \in \Gamma}\left(\lambda_{i}(X)+\tau\right) / \alpha-\rho \\
-\min _{i \in \Gamma} \lambda_{i}(X) / \alpha \stackrel{(77)}{=}
\end{array}
$$

If $s<r$, we have

$$
\begin{array}{r}
\underline{\lambda}_{D}=-\max _{i \in \Gamma^{\perp}} \lambda_{i}\left(X^{\prime}\right) / \alpha-\rho \stackrel{(81)}{=}-\max _{i \in \boldsymbol{b}^{\prime} \cup \boldsymbol{c}^{\prime}} \lambda_{i}\left(X^{\prime}\right) / \alpha-\rho \\
\stackrel{(76)}{=} \\
-\lambda_{r}\left(X^{\prime}\right) / \alpha-\rho \stackrel{(76)}{\geq}
\end{array}
$$


Thus, the proof is completed.

Remark 5.1 We would like to describe the relation between an $F$-stationary point and an $\alpha$-stationary point based on Theorems 5.1, 5.2 and 5.3.

(i) When $s<r$, for each $\mathcal{C}$, one can observe that an $F$-stationary point is the same as an $\alpha$-stationary point. For example, from (71) and (72), when $s<r, \underline{\lambda}_{D} \geq-\lambda_{r}(X) / \alpha=0$ due to $X \in \mathcal{C}_{3}$, then $D \in \mathbb{S}_{+}^{n-s}$. When $s=r$, an $\alpha$-stationary point must be an $F$-stationary point, but the converse is not true. Therefore, being an $F$-stationary point is a weaker necessary optimality condition than is being an $\alpha$-stationary point.

(ii) The $\alpha$-stationary point as a stronger necessary condition has been widely studied in $[11,28,30,45,47,49]$, and under some mild conditions, a global optimal solution is an $\alpha$-stationary point for some $\alpha>0$; see [47, Theorem 2.2] or Theorem 6.1 (1) established in the next section. Therefore, in terms of guaranteeing an optimal solution, being an $\alpha$-stationary point is a better condition than being an $F$-stationary point.

(iii) Most importantly, compared with that of an $F$-stationary point, the characterization of an $\alpha$-stationary point presents a much easier way to design numerical algorithms to search. The updating scheme usually takes the form

$$
X^{k+1}=\Pi_{\mathbb{S}^{n}(r) \cap \mathcal{C}}\left(X^{k}-\alpha \nabla f\left(X^{k}\right)\right)
$$

based on $\alpha$-stationary point (43), where $X^{k}$ is the currently computed iterate; see $[11,28,47,49]$ for more details. Numerical experiments have demonstrated that such a scheme could yield desirable solutions with fast computational speed since the projection $\Pi_{\mathbb{S}^{n}(r) \cap \mathcal{C}}\left(X^{k}-\alpha \nabla f\left(X^{k}\right)\right)$ often has a closed form, such as the cases of $\mathcal{C}=\mathcal{C}_{i}, i=1,2,3$.

\section{Local and Global Minimizers}

Given the expressions presented in the previous section, this section establishes the relationships among the stationary points and the global/local minimizers of (1). We first introduce the so-called $r$-restricted subspace.

Definition 6.1 Let $X \in \mathbb{S}^{n}(r)$ with $s:=\operatorname{rank}(X)$ have the same EVD as in (6). We call the subspace

$$
\mathbb{S}_{X}^{n}:=\left\{\mathcal{M}_{X}(A, 0): A \in \mathbb{S}^{s}\right\}
$$

the $X$-restricted subspace. In fact, $X \in \mathbb{S}_{X}^{n} \subseteq \mathbb{S}^{n}(r)$.

We say the gradient of a function $f$ is Lipschitz continuous with constant $L_{f}$ over $\mathbb{S}^{n}$ if

$$
\|\nabla f(X)-\nabla f(Y)\| \leq L_{f}\|X-Y\|, \quad \forall X, Y \in \mathbb{S}^{n} .
$$

Our first result is to analyze the relations between an $\alpha$-stationary point and the local/global minimizers of (1). 
Theorem 6.1 For problem (1), the relations between an $\alpha$-stationary point and the local and global minimizers are given as follows.

(A) If the gradient $\nabla f$ is Lipschitz with constant $L_{f}$ over $\mathbb{S}^{n}$, then a global minimizer of (1) is also an $\alpha$-stationary point for any $0<\alpha<1 / L_{f}$.

(B) Let $f$ be convex and $X \in \mathbb{S}^{n}(r) \cap \mathcal{C}$ be an $\alpha$-stationary point.

(B1) If $X \in \mathrm{bdC}$, then $X$ is a global minimizer of (1) restricted on $\mathbb{S}_{X}^{n}$.

(B2) If $X \in \operatorname{intC}$ with $\operatorname{rank}(\mathrm{X})=\mathrm{r}$, then $X$ is a global minimizer of (1) restricted on $\mathbb{S}_{X}^{n}$.

(B3) If $X \in \operatorname{int} \mathcal{C}$ with $\operatorname{rank}(\mathrm{X})<\mathrm{r}$, then $X$ is a global minimizer of (1).

Proof. (A) This conclusion is proven in [47, Theorem 2.2] for the vector case. For the sake of completeness, we provide the proof of this part. Let $X$ be a minimizer of (1). Suppose that there exists a matrix $Y$,

$$
X \neq Y \in \Pi_{\mathbb{S} n}(r) \cap \mathcal{C}(X-\alpha \nabla f(X)) .
$$

It follows that

$$
\|Y-X+\alpha \nabla f(X)\|_{F}^{2} \leq\|X-X+\alpha \nabla f(X)\|_{F}^{2},
$$

which suffices to show that

$$
\langle\nabla f(X), Y-X\rangle \leq-(1 /(2 \alpha))\|Y-X\|^{2} .
$$

Since $f$ is the gradient Lipschitz with a positive Lipschitz constant $L_{f}$ and $0<\alpha<1 / L_{f}$, by the Descent Lemma [50, Proposition A.24] and (84), we have

$$
\begin{aligned}
f(Y) & \leq f(X)+\langle\nabla f(X), Y-X\rangle+\frac{L_{f}}{2}\|Y-X\|^{2} \\
& \leq f(X)+\frac{L_{f}-1 / \alpha}{2}\|Y-X\|^{2}<f(X),
\end{aligned}
$$

contradicting the optimality of $X$.

(B1) We consider three cases:

Case of $\mathcal{C}=\mathcal{C}_{1}$. If $X$ is an $\alpha$-stationary point, then from Theorem 5.1, there exists a $\rho \leq 0$ and a $D \in \mathbb{S}^{n-s}$ such that

$$
\nabla f(X)=\mathcal{M}_{X}\left(\rho \Lambda_{\Gamma}(X), D\right) .
$$

Since $X \in \mathrm{bdC}_{1},\|X\|_{F}=\left\|\Lambda_{\Gamma}(X)\right\|_{F}=1$. For any $Y \in \mathcal{C}_{1} \cap \mathbb{S}_{X}^{n}$, there exists an $A \in \mathbb{S}^{s}$ with $\|A\|_{F} \leq 1$ such that $Y=\mathcal{M}_{X}(A, 0)$, which yields

$$
Y-X=\mathcal{M}_{X}(A, 0)-\mathcal{M}_{X}\left(\Lambda_{\Gamma}(X), 0\right)=\mathcal{M}_{X}\left(A-\Lambda_{\Gamma}(X), 0\right) .
$$

For any $Y \in \mathbb{S}_{X}^{n} \cap \mathcal{C}_{1}, X+Y \in \mathbb{S}^{n}(r)$. As $f$ is convex, it holds that

$$
\begin{aligned}
f(Y)-f(X) & \geq\langle\nabla f(X), Y-X\rangle \\
& =\left\langle\mathcal{M}_{X}\left(\rho \Lambda_{\Gamma}(X), D\right), \mathcal{M}_{X}\left(A-\Lambda_{\Gamma}(X), 0\right)\right\rangle \\
& =\rho\left\langle\Lambda_{\Gamma}(X), A-\Lambda_{\Gamma}(X)\right\rangle=\rho\left\langle\Lambda_{\Gamma}(X), A\right\rangle-\rho \\
& \geq \rho\|A\|_{F}\left\|\Lambda_{\Gamma}(X)\right\|_{F}-\rho \geq 0 .
\end{aligned}
$$


Therefore, $X$ is a global minimizer of (1) restricted on $\mathbb{S}_{X}^{n}$.

Case of $\mathcal{C}=\mathcal{C}_{2}$. If $X$ is an $\alpha$-stationary point, then from Theorem 5.2, there exists an $A \in \mathbb{S}_{-}^{\left|\boldsymbol{\eta}_{+}\right|}, C \in \mathbb{S}_{+}^{\left|\boldsymbol{\eta}_{-}\right|}, D \in \mathbb{S}^{\left|\boldsymbol{\eta}_{=}\right|}$, where $\boldsymbol{\eta}_{+}, \boldsymbol{\eta}_{ \pm}, \boldsymbol{\eta}_{-}, \boldsymbol{\eta}_{=}$are defined as in (37), such that

$$
\nabla f(X)=\mathcal{N}_{X}(A, 0, C, D) .
$$

For any $Y \in \mathbb{S}_{X}^{n} \cap \mathcal{C}_{2}$, it follows that

$$
\begin{aligned}
Y-X & =\mathcal{N}_{X}\left(A_{1}, A_{2}, A_{3}, 0\right)-\mathcal{N}_{X}\left(t I_{\left|\boldsymbol{\eta}_{+}\right|}, A_{\boldsymbol{\eta}_{ \pm}}(X),-t I_{\left|\boldsymbol{\eta}_{-}\right|}, 0\right) \\
& =\mathcal{N}_{X}\left(A_{1}-t I_{\left|\boldsymbol{\eta}_{+}\right|}, A_{2}-\Lambda_{\boldsymbol{\eta}_{ \pm}}(X), A_{3}+t I_{\left|\boldsymbol{\eta}_{-}\right|}, 0\right) .
\end{aligned}
$$

By $Y \in \mathcal{C}_{2}$, it has $A_{1}-t I_{\left|\boldsymbol{\eta}_{+}\right|} \in \mathbb{S}_{-}^{\left|\boldsymbol{\eta}_{+}\right|}$and $A_{3}+t I_{\left|\boldsymbol{\eta}_{+}\right|} \in \mathbb{S}_{+}^{\left|\boldsymbol{\eta}_{-}\right|}$. These results suffice to show that

$$
\langle\nabla f(X), Y-X\rangle=\left\langle A, A_{1}-t I_{\left|\boldsymbol{\eta}_{+}\right|}\right\rangle+\left\langle C, A_{3}+t I_{\left|\boldsymbol{\eta}_{-}\right|}\right\rangle \geq 0,
$$

which together with the convexity of $f$ yields the conclusion.

Case of $\mathcal{C}=\mathcal{C}_{3}$. If $X$ is an $\alpha$-stationary point, then from Theorem 5.3, there exists a $\rho \in \mathbb{R}$ and $D \in \mathbb{S}^{n-s}$ such that

$$
\nabla f(X)=\mathcal{M}_{X}\left(\rho I_{s}, \rho I_{n-s}+D\right) .
$$

Since $X \in \operatorname{bd} \mathcal{C}_{3}$, then $\left\langle I_{s}, \Lambda_{\Gamma}(X)\right\rangle=1$. For any $Y \in \mathcal{C}_{3} \cap \mathbb{S}_{X}^{n}$, there exists an $A \in \mathbb{S}^{s}$ with $\left\langle I_{s}, A\right\rangle=1$ such that $Y=\mathcal{M}_{X}(A, 0)$, which yields

$$
Y-X=\mathcal{M}_{X}(A, 0)-\mathcal{M}_{X}\left(\Lambda_{\Gamma}(X), 0\right)=\mathcal{M}_{X}\left(A-\Lambda_{\Gamma}(X), 0\right) .
$$

For any $Y \in \mathbb{S}_{X}^{n} \cap \mathcal{C}_{3}, X+Y \in \mathbb{S}^{n}(r)$. As $f$ is convex, it holds that

$$
\begin{aligned}
f(Y)-f(X) & \geq\langle\nabla f(X), Y-X\rangle \\
& =\left\langle\mathcal{M}_{X}\left(\rho I_{s}, \rho I_{n-s}+D\right), \mathcal{M}_{X}\left(A-\Lambda_{\Gamma}(X), 0\right)\right\rangle \\
& =\rho\left\langle I_{s}, A-\Lambda_{\Gamma}(X)\right\rangle=\rho(1-1)=0 .
\end{aligned}
$$

Therefore, $X$ is a global minimizer of (1) restricted on $\mathbb{S}_{X}^{n}$.

(B2) and (B3) If $X \in \operatorname{int} C$, problem (1) reduces to rank-constrained optimization. These results have been established by [30, Theorem 2] and are omitted here.

Our next major result is to analyze the relations between an $F$-stationary point and the local/global minimizers of (1).

Theorem 6.2 For problem (1), the relations between an F-stationary point and the local and global minimizers are given as follows.

(A) A local optimal solution of (1) is also an F-stationary point.

(B) Let $f$ be convex and $X \in \mathbb{S}^{n}(r) \cap \mathcal{C}$ be an $F$-stationary point.

(B1) If $X \in \mathrm{bdC}$, then $X$ is a global minimizer of (1) restricted on $\mathbb{S}_{X}^{n}$.

(B2) If $X \in \operatorname{intC}$ with $\operatorname{rank}(\mathrm{X})=\mathrm{r}$, then $X$ is a global minimizer of (1) restricted on $\mathbb{S}_{X}^{n}$.

(B3) If $X \in \operatorname{int} \mathcal{C}$ with $\operatorname{rank}(\mathrm{X})<\mathrm{r}$, then $X$ is a global minimizer of (1). 
Proof. (A) If $X$ is a local optimal solution of (1), then $0 \in \nabla f(X)+N_{\mathbb{S}^{n}(r) \cap \mathcal{C}}^{F}(X)$ by [26, Theorem 6.12]. From Theorem 4.1, $0 \in \nabla f(X)+N_{\mathbb{S}^{n}(r)}^{F}(X)+N_{\mathcal{C}}^{F}(X)$, namely, $X$ is an $F$-stationary point.

(B) The proof is omitted here since it is similar to that of (B) in Theorem 6.1.

To end this section, we bring an example to illustrate Theorem 6.1.

Example 6.1 Consider the problem

$$
\begin{aligned}
& \min _{X \in \mathbb{S}^{3}} f(X) \quad:=\frac{1}{2}\|X-Y\|_{F}^{2} \\
& \text { s.t. } \quad \operatorname{rank}(X) \leq 2 \\
& \quad\|X\|_{F} \leq 1
\end{aligned}
$$

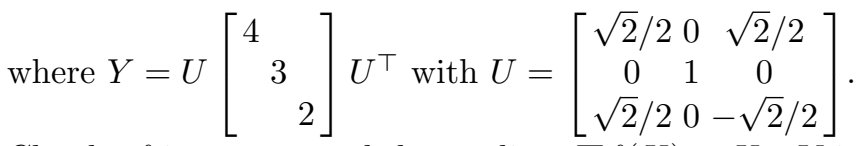

Clearly, $f$ is convex, and the gradient $\nabla f(X)=X-Y$ is Lipschitz continuous with constant $L_{f}=1$ over $\mathbb{S}^{3}$. Based on (17), the global minimizer of (85) is

$$
X^{*}:=\Pi_{\mathbb{S}^{3}(2) \cap \mathcal{C}_{1}}(Y)=U \operatorname{Diag}(4 / 5,3 / 5,0) U^{\top} .
$$

Direct calculation yields $\nabla f\left(X^{*}\right)=X^{*}-Y=-U \operatorname{Diag}(16 / 5,12 / 5,2) U^{\top}$ and $X^{*} \in \operatorname{bd} \mathcal{C}_{1}$. From (45), we have

$$
\begin{aligned}
& \Theta_{\rho}\left(X^{*}\right):=\left\{\mathcal{M}_{X^{*}}\left(\rho \Lambda_{\Gamma}\left(X^{*}\right), D\right): \begin{array}{l}
\rho \leq 0, D \in \mathbb{S}^{3-2} \\
\bar{\lambda}_{D} \leq\left(\frac{1}{\alpha}-\rho\right) \lambda_{2}^{\mathrm{abs}}\left(X^{*}\right)
\end{array}\right\} \\
& =\left\{U\left[\begin{array}{lll}
4 \rho / 5 & & \\
& 3 \rho / 5 & \\
& & D
\end{array}\right] U^{\top}: \begin{array}{l}
\rho \leq 0, D \in \mathbb{R} \\
\end{array}\right.
\end{aligned}
$$

Then, it can be seen that $\nabla f\left(X^{*}\right) \in \Theta_{\rho}\left(X^{*}\right)$ with $\rho=-4$ for any $\alpha>0$, which together with Theorem 5.1 (ii), reveals that $X^{*}$ is an $\alpha$-stationary point of (85).

Let us consider a point $\bar{X}:=U \operatorname{Diag}(2 / \sqrt{5}, 0,1 / \sqrt{5}) U^{\top}$. Direct calculation gives $\nabla f(\bar{X})=U \operatorname{Diag}(2 / \sqrt{5}-4,-3,1 / \sqrt{5}-2) U^{\top}$ and

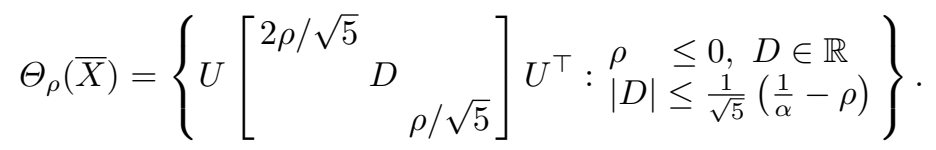

Then, it holds that $\nabla f(\bar{X}) \in \Theta_{\rho}(\bar{X})$ with $\rho=1-2 \sqrt{5}$ for any $0<\alpha \leq \frac{1}{\sqrt{5}+1}$, which together with Theorem 5.1 (ii), indicates that $X^{*}$ is also an $\alpha$-stationary point with $0<\alpha \leq \frac{1}{\sqrt{5}+1}$ of (85). Apparently, $\bar{X}$ is not the global minimizer of (85), but it is a global minimizer restricted on

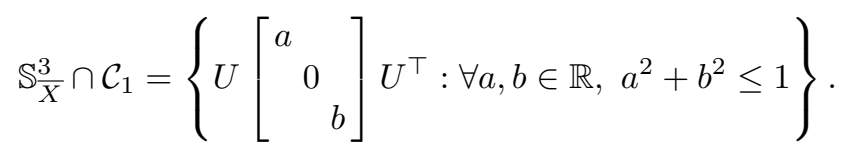




\section{Conclusions}

In this paper, with the help of explicit expressions of the projections and Fréchet normal cones to constrained sets, we have established the first-order optimality conditions (i.e., $\alpha$-stationary point and $F$-stationary point) of the MOLS and have revealed the relationship between each stationary point and each local/global minimizer of the MOLS. Whether similar results hold for low-rank optimization problems with general constraints, such as linear/nonlinear equalities and inequalities, remains to be determined. We leave this topic to be investigated in the future.

Acknowledgements This work was supported in part by the National Natural Science Foundation of China (11431002).

\section{References}

1. Recht, B., Fazel, M., Parrilo, P.A.: Guaranteed minimum-rank solutions of linear matrix equations via nuclear norm minimization. SIAM Review 52(3), 471-501 (2010)

2. Markovsky, I.: Low Rank Approximation. Springer, London (2012)

3. Ding, C.: An introduction to a class of matrix optimization problems. Ph.D. thesis, National University of singapore (2012)

4. Udell, M., Horn, C., Zadeh, R.B., Boyd, S.P.: Generalized low rank models. Machine Learning 9(1), 1-118 (2016)

5. Davenport, M.A., Romberg, J.: An overview of low-rank matrix recovery from incomplete observations. IEEE Journal of Selected Topics in Signal Processing 10(4), 608-622 (2016)

6. Fazel, M.: Matrix rank minimization with applications. Ph.D. thesis, Stanford University (2002)

7. Candès, E.J., Tao, T.: The power of convex relaxation: Near-optimal matrix completion. IEEE Transactions on Information Theory 56(5), 2053-2080 (2010)

8. Marjanovic, G., Solo, V.: On $l_{q}$ optimization and matrix completion. IEEE Transactions on Signal Processing 60(11), 5714-5724 (2012)

9. Mohan, K., Fazel, M.: Iterative reweighted algorithms for matrix rank minimization. Journal of Machine Learning Research 13(Nov), 3441-3473 (2012)

10. Nie, F., Wang, H., Cai, X., Huang, H., Ding, C.: Robust matrix completion via joint schatten p-norm and $l_{p}$-norm minimization. In: IEEE International Conference on Data Mining, pp. 566-574 (2012)

11. Chen, Y., Xiu, N., Peng, D.: Global solutions of non-lipschitz $s_{2}-s_{p}$ minimization over the positive semidefinite cone. Optimization Letters 8(7), 2053-2064 (2014)

12. Zhao, Y.B.: An approximation theory of matrix rank minimization and its application to quadratic equations. Linear Algebra and Its Applications 437(1), 77-93 (2012)

13. Yao, H., Debing, Z., Jieping, Y., Xuelong, L., Xiaofei, H.: Fast and accurate matrix completion via truncated nuclear norm regularization. IEEE Transactions on Pattern Analysis and Machine Intelligence 35(9), 2117-2130 (2013)

14. Burer, S., Monteiro, R.D.C.: A nonlinear programming algorithm for solving semidefinite programs via low-rank factorization. Mathematical Programming 95(2), 329-357 (2003)

15. Journée, M., Bach, F., Absil, P.A., Sepulchre, R.: Low-rank optimization on the cone of positive semidefinite matrices. SIAM Journal on Optimization 20(5), 2327-2351 (2010)

16. Wen, Z., Yin, W., Zhang, Y.: Solving a low-rank factorization model for matrix completion by a nonlinear successive over-relaxation algorithm. Mathematical Programming Computation 4(4), 333-361 (2012) 
17. Jain, P., Netrapalli, P., Sanghavi, S.: Low-rank matrix completion using alternating minimization. In: Proceedings of the forty-fifth annual ACM symposium on Theory of computing, pp. 665-674. ACM (2013)

18. Hardt, M.: Understanding alternating minimization for matrix completion. In: Foundations of Computer Science (FOCS), 2014 IEEE 55th Annual Symposium on, pp. 651-660. IEEE (2014)

19. Sun, R., Luo, Z.: Guaranteed matrix completion via non-convex factorization. IEEE Transactions on Information Theory 62(11), 6535-6579 (2016)

20. Gao, Y.: Structured low rank matrix optimization problems: A penalty approach. Ph.D. thesis, National University of singapore (2010)

21. Kim, S.J., Moon, Y.H.: Structurally constrained $H_{2}$ and $H_{\infty}$ control: a rank-constrained LMI approach. Automatica 42(9), 1583-1588 (2006)

22. Delgado, R.A., Agüero, J.C., Goodwin, G.C.: A rank-constrained optimization approach: Application to factor analysis. IFAC Proceedings Volumes 47(3), 10,373-10,378 (2014)

23. Bi, S., Pan, S.: Error bounds for rank constrained optimization problems and applications. Operations Research Letters 44(3), 336-341 (2016)

24. Zhou, S., Xiu, N., Qi, H.: A fast matrix majorization-projection method for penalized stress minimization with box constraints. IEEE Transactions on Signal Processing 66(16), 4331-4346 (2018)

25. Luke, D.R.: Prox-regularity of rank constraint sets and implications for algorithms. Journal of Mathematical Imaging and Vision 47(3), 231-238 (2013)

26. Rockafellar, R.T., Wets, R.J.: Variational Analysis. Springer (1998)

27. Cason, T.P., Absil, P.A., Dooren, P.V.: Iterative methods for low rank approximation of graph similarity matrices. Linear Algebra and Its Applications 438(4), 1863-1882 (2013)

28. Schneider, R., Uschmajew, A.: Convergence results for projected line-search methods on varieties of low-rank matrices via łojasiewicz inequality. SIAM Journal on Optimization 25(1), 622-646 (2015)

29. Zhou, G., Huang, W., Gallivan, K.A., Van Dooren, P., Absil, P.A.: A Riemannian rankadaptive method for low-rank optimization. Neurocomputing 192, 72-80 (2016)

30. Li, X., Song, W., Xiu, N.: Optimality conditions for rank-constrained matrix optimization. Journal of the Operations Research Society of China 7(2), 285-301 (2019)

31. Linial, N., London, E., Rabinovich, Y.: The geometry of graphs and some of its algorithmic applications. Combinatorica 15(2), 215-245 (1995)

32. Biswas, P., Ye, Y.: Semidefinite programming for ad hoc wireless sensor network localization. In: International Symposium on Information Processing in Sensor Networks (2004)

33. Ji, S., Sze, K.F., Zhou, Z., So, M.C., Ye, Y.: Beyond convex relaxation: A polynomialtime non-convex optimization approach to network localization. In: IEEE Infocom (2013)

34. Borsdorf, R., Higham, N.J., Raydan, M.: Computing a nearest correlation matrix with factor structure. SIAM Journal on Matrix Analysis and Applications 31(5), 2603-2622 (2010)

35. Higham, N.J.: Computing the nearest correlation matrix a problem from finance. Ima Journal of Numerical Analysis 22(3), 329-343 (2018)

36. Dukanovic, I., Rendl, F.: Semidefinite programming relaxations for graph coloring and maximal clique problems. Mathematical Programming 109(2-3), 345-365 (2007)

37. Kalev, A., Kosut, R.L., Deutsch, I.H.: Quantum tomography protocols with positivity are compressed sensing protocols. Nature Partner Journals Quantum Information 1(1), $15,018(2015)$

38. Lewis, A.S.: Group invariance and convex matrix analysis. SIAM Journal on Matrix Analysis and Applications 17(4), 927-949 (1996)

39. Tam, M.K.: Regularity properties of non-negative sparsity sets. Journal of Mathematical Analysis and Applications 447(2), 758-777 (2017)

40. Lu, Z., Zhang, Y., Li, X.: Penalty decomposition methods for rank minimization. Optimization Methods and Software 30(3), 531-558 (2015) 
41. Kyrillidis, A.: Rigorous optimization recipes for sparse and low rank inverse problems with applications in data sciences. Ph.D. thesis, École Polytechnique Fédérale de Lausanne (2014)

42. Mordukhovich, B.S.: Variational analysis and generalized differentiation I: Basic theory, vol. 330. Springer Science and Business Media (2006)

43. Hiriart-Urruty, J.B., Lemaréchal, C.: Fundamentals of convex analysis. Springer Science and Business Media (2012)

44. Drusvyatskiy, D., Kempton, C.: Variational analysis of spectral functions simplified. Journal of Convex Analysis 25(1), 119-134 (2018)

45. Lu, Z.: Optimization over sparse symmetric sets via a nonmonotone projected gradient method. arXiv preprint arXiv:1509.08581, (2015)

46. Pan, L., Xiu, N., Fan, J.: Optimality conditions for sparse nonlinear programming. Science China Mathematics 60(5), 1-18 (2017)

47. Beck, A., Eldar, Y.C.: Sparsity constrained nonlinear optimization: Optimality conditions and algorithms. SIAM Journal on Optimization 23(3), 1480-1509 (2013)

48. Horn, R.A., Johnson, C.R.: Matrix analysis, Second Ed. Cambriddge University Press, New York (2013)

49. Pan, L., Zhou, S., Xiu, N., Qi, H.D.: A convergent iterative hard thresholding for nonnegative sparsity optimization. Pacific Journal of Optimization 13(2), 325-353 (2017)

50. Bertsekas, D.P.: Nonlinear Programming: Second Ed. Athena Scientific, Belmont (1999) 